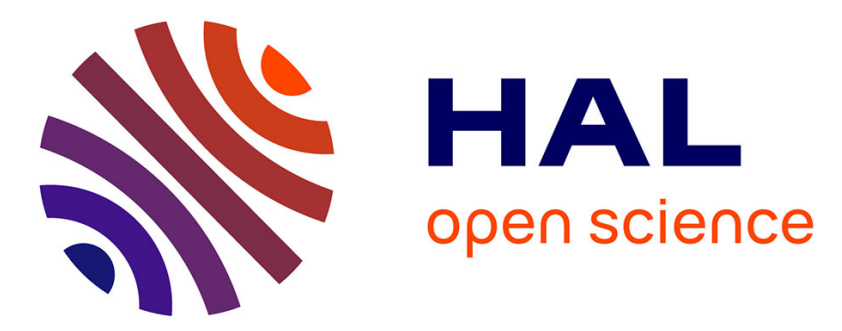

\title{
A new estimation of equivalent matrix block sizes in fractured media with two-phase flow applications in dual porosity models
}

Chahir Jerbi, André Fourno, Benoit Noetinger, Frédérick Delay

\section{- To cite this version:}

Chahir Jerbi, André Fourno, Benoit Noetinger, Frédérick Delay. A new estimation of equivalent matrix block sizes in fractured media with two-phase flow applications in dual porosity models. Journal of Hydrology, 2017, 548, pp.508-523. 10.1016/j.jhydrol.2017.03.028 . hal-01738345

\section{HAL Id: hal-01738345 \\ https: / hal-ifp.archives-ouvertes.fr/hal-01738345}

Submitted on 20 Mar 2018

HAL is a multi-disciplinary open access archive for the deposit and dissemination of scientific research documents, whether they are published or not. The documents may come from teaching and research institutions in France or abroad, or from public or private research centers.
L'archive ouverte pluridisciplinaire HAL, est destinée au dépôt et à la diffusion de documents scientifiques de niveau recherche, publiés ou non, émanant des établissements d'enseignement et de recherche français ou étrangers, des laboratoires publics ou privés. 
4

\title{
A new estimation of equivalent matrix block sizes in fractured media with two-phase
} flow applications in dual porosity models

\author{
Chahir Jerbi ${ }^{1}$, André Fourno ${ }^{1}$, Benoit Noetinger ${ }^{1}$, Frederick Delay ${ }^{2 *}$ \\ ${ }^{1}$ IFP Energies Nouvelles, 1\&4 Avenue du Bois Préau 92500 Rueil-Malmaison, France. \\ ${ }^{2}$ Laboratoire d'Hydrologie et de Géochimie de Strasbourg, Univ. Strasbourg/EOST, CNRS \\ UMR 7517, 1 rue Blessig, 67000 Strasbourg, France \\ * Corresponding author: Phone +33 3688504 16; Fax +33 3688504 02; mail fdelay@ unistra.fr
}

\section{Abstract}

Single and multiphase flows in fractured porous media at the scale of natural reservoirs are often handled by resorting to homogenized models that avoid the heavy computations associated with a complete discretization of both fractures and matrix blocks. For example, the two overlapping continua (fractures and matrix) of a dual porosity system are coupled by way of fluid flux exchanges that deeply condition flow at the large scale. This characteristic is a key to realistic flow simulations, especially for multiphase flow as capillary forces and contrasts of fluid mobility compete in the extraction of a fluid from a capacitive matrix then conveyed through the fractures. The exchange rate between fractures and matrix is conditioned by the so-called mean matrix block size which can be viewed as the size of a single matrix block neighboring a single fracture within a mesh of a dual porosity model.

We propose a new evaluation of this matrix block size based on the analysis of discrete fracture networks. The fundaments rely upon establishing at the scale of a fractured block the equivalence between the actual fracture network and a Warren and Root network only made of three regularly spaced fracture families parallel to the facets of the fractured block. The resulting matrix block sizes are then compared via geometrical considerations and 
26 two-phase flow simulations to the few other available methods. It is shown that the new

27 method is stable in the sense it provides accurate sizes irrespective of the type of fracture 28 network investigated. The method also results in two-phase flow simulations from dual 29 porosity models very close to that from references calculated in finely discretized networks.

30 Finally, calculations of matrix block sizes by this new technique reveal very rapid, which 31 opens the way to cumbersome applications such as preconditioning a dual porosity approach 32 applied to regional fractured reservoirs.

\section{Keywords}

35 Fractured porous media; Matrix block sizes; Dual porosity models; Multiphase flow. 


\section{Introduction}

Conventional oil reservoirs are often housed in fractured rocks, especially in carbonates environments, and one can estimate that more than $30 \%$ of world oil reserves are concealed in densely fractured systems, oil being mainly trapped in the host rock matrix. Paradoxically, these geological structures may trigger contrasted effects on large-scale twophase flow patterns by increasing oil recovery due to high local permeability values, or on the opposite, by decreasing oil extraction rates because of early water invasion, viscous fingering etc. The same type of behavior is also encountered in the context of water decontamination and can become even more complex if oil (and/or water) is swept by injections of miscible gas.

Modeling two-phase flow in fractured reservoirs is now often employed for the purpose of various applications, for instance to assess the relevance of different oil recovery strategies or to investigate on the feasibility of in-situ water decontamination processes (Bourbiaux, 2010). This fact makes that modeling single phase or multiphase flow in fractured media is still a fertile research domain even though pioneering works on the topic started in the early sixties (e.g., in Lemonnier et al., 2010a, b).

In this context, flow simulations relying upon finely gridded discrete fracture networks and their associated (discretized) matrix blocks are becoming increasingly popular because of the availability of high performance computers, the progress in algorithms for meshing complex geometries, and the availability of sophisticated numerical techniques for solving partial differential equations (Landereau et al., 2001; Noetinger et al., 2001; Adler et al., 2005; Matthäi and Nick, 2009; Fourno et al., 2013). This exhaustive approach is critical to bring us reference solutions and various benchmarks with which simpler approaches can be compared. Nevertheless, gridded discrete fracture networks may be poorly documented and include flawed information in the case of real-world applications. In addition, finely gridded 
systems remain hardly usable for current practical applications to large-scale systems that result in cumbersome model parameterizations and heavy computations. This downside is emphasized in the domain of petroleum engineering usually dealing with both non-linear multiphase flow and dense fracture networks requiring huge discretization efforts (Landereau et al., 2001; Adler et al., 2005; Fourno et al., 2013). Applicability is also hindered by duplicated calculations if the study encompasses tests of various model designs, various model parameterization and various flow scenarios.

Fortunately, dense fracture networks are also good candidates to homogenization at the scale of reasonable elementary mesh sizes (on the order of 5-100 m) by resorting for example to the dual porosity approach to fractured media initially developed by Barenblatt et al. (1960). The dual porosity formulation conceptualizes a fractured system as two overlapping continua merging a fracture medium and a matrix medium with contrasts of hydraulic properties between the two continua. Flow is then described by a set of equations in each continuum (this set depends on the type of flow and the fluid phases present in the system) associated with an exchange term ruling the fluid fluxes percolating between continua.

This exchange term is all the more important that in general fractures are conveying flow as the matrix stores fluid volumes. In transient problems as for example forced flow between injecting and extracting wells, the way the relationship establishes between storage capacity and conduction property conditions the overall response of the reservoir (e.g., Acuna and Yortsos, 1995). In the specific cases of two-phase flow (water and oil), the absence or the weak incidence of capillary forces in open fractures makes that flow is locally mainly of single-phase type conveying either oil or water (with sharp saturation fronts in between) at different locations in the fracture network. For its part, the way the matrix blocks are soaked (water invades the matrix and pushes oil away) or drained (oil pushes water) strongly depends 
on matrix block sizes and on the petro-physics properties of the matrix, making that extraction from the matrix of a fluid by the other is mainly driven by capillary forces or by capillary forces plus viscous forces (single-phase Darcian flow to make it simple).

When a discrete fracture network is connected enough and handled at an elementary scale larger than a representative elementary volume, the exchange term in the dual porosity models is proportional to an equivalent matrix block size. Intuitively, a REV for a fracture network is a volume within which mean properties of the network such as fracture density, fracture aperture, fracture hydraulic conductivity have some statistical meaning (Long et al., 1982; Neuman, 1988). In a dual porosity model, the REV is also associated with the capability to represent the actual fracture network as a synthetic network made (in threedimensional problems) of three regularly spaced fracture families, each family developing fracture planes normal to one of the three main directions of flow. The so-called DFN homogenized as a "sugar-cube" model (Warren and Root, 1963) is at the origin of the notion of the equivalent matrix block size in relation with the dimensions of the elementary "sugar piece" separating neighbor fractures in the homogenized DFN (Kazemi et al., 1976).

There exist two types of methods to evaluate the elementary matrix block size. The first type relies upon exercises matching actual well test drawdown curves with analytical solutions that inherit from rigorous mathematical homogenization or large-scale averaging techniques (Arbogast 1990; Quintard and Whitaker, 1993; Noetinger et al., 2001; Unsal et al., 2010; Noetinger and Jarrige, 2012). The downside of these techniques is that sometimes actual well testing in fractured rock do not exist and when these tests exist, the damaged zone in the close vicinity of a well may not fully reflect flow conditions in the natural fracture network. The second type of methods is based on geometrical considerations regarding the fracture network. These considerations led to three geometrical approaches that are the geometrical imbibition method (GI, Bourbiaux et al., 1997), the enhanced general imbibition 
111 method (EGI, Bourbiaux et al., 2006), and the mean spacing method (MS, Narr, 1996). It is

112 obvious that these approaches can only be applied if a minimum prior knowledge about the 113 fracture network geometry is available.

114 In this contribution we propose a new geometrical method that can to some extent 115 overlook the actual geometry of the fracture network because the method relies upon the 116 identification of a sugar-cube DFN equivalent to the actual network (see details hereafter).

117 The method also allows us to calculate matrix block sizes along directions parallel to the main 118 flow directions that are conditioned by the geometry of the fracture network (or its equivalent 119 as a sugar-cube model). Section 2 (and Appendix A) is focused on the theoretical framework 120 we rely upon to build the so-called oriented block size (OBS) method that we propose. For the 121 sake of clarity, a few features about dual-porosity models are also reminded. The matrix block 122 sizes stemming from the OBS technique are then compared to that from the other geometrical 123 techniques (GI, EGI, and MS, see above). The comparison is performed by way of a suite of 124 calculations applied to synthetic random fracture networks for which we explicitly control 125 both the geometric and hydraulic properties of the networks and the mean size of matrix 126 blocks between fractures. As told earlier, only dense and well-connected fracture networks are 127 considered because sparse networks cannot be homogenized via a dual porosity model at the 128 scale of a complete underground reservoir. Section 4 evaluates the OBS technique and also 129 the other geometrical approaches within the framework of a dual-porosity model compared 130 with exhaustive calculations discretizing the fracture network and the matrix blocks. The two131 phase flow scenarios are either dominated by capillary forces or viscous forces in an exercise 132 which consists in draining oil from matrix blocks by injecting water in fractures. These 133 complex flow scenarios are performed over synthetic test cases in which we control the 134 reference calculations (in a fully discretized system). This procedure enable us to clearly 
135 emphasizes the main theoretical findings regarding matrix block size in dual porosity models

136 before envisioning further concrete field-scale applications.

\section{Theoretical background}

In various approaches to fractured systems, the duality of fracture networks embedded

140 in a host rock matrix is often represented as two overlapping continua merging a fracture

141 medium and a matrix medium. In a so-called dual porosity - single permeability model, the

142 fractures are usually highly conductive and poorly capacitive as the matrix is highly

143 capacitive but with negligible flow triggered by fluid pressure gradients (weak permeability).

144 As an example, single-phase Darcian flow in a dual continuum approach results in the

145 resolution of two equations in the form

$146 \frac{\partial\left(\rho \phi^{f}\right)}{\partial t}+\nabla \cdot\left(-\rho \frac{\mathbf{k}^{f}}{\mu} \cdot \nabla\left(P^{f}+\rho g z\right)\right)-E^{m \rightarrow f}=0$

$\frac{\partial\left(\rho \phi^{m}\right)}{\partial t}+E_{p}^{m \rightarrow f}=0 ; E_{p}^{m \rightarrow f}=\rho \boldsymbol{\sigma} \frac{\mathbf{k}^{m}}{\mu}\left(P^{m}-P^{f}\right)$

148 For the sake of simplicity, references to space $(\mathrm{x})$ and time (t) for parameters and state

149 variables have been dropped. The indexes $\mathrm{f}$ and $\mathrm{m}$ refer to fracture and matrix continua,

150 respectively. $\rho_{[\mathrm{ML}-3]}$ is the mass density of the fluid, $\mu$ [ML-1T-1] is the dynamic

151 viscosity of the fluid, $\mathbf{k}^{\lambda}$ [L2] is the permeability of the continuum $\lambda(\lambda=f, m), \phi^{\lambda}$ [-] is

152 the porosity of the continuum $\lambda, P^{\lambda}$ [ML-1T-2] is the fluid pressure in the continuum $\lambda, \mathrm{g}$

153 [LT-2] is the scalar value of the gravity acceleration $\mathrm{g}$, and $\mathrm{z}[\mathrm{L}]$ is the local elevation taken

154 from an arbitrary reference and counted positive upward. $E_{p}^{m \rightarrow f}\left[\mathrm{ML}^{-3} \mathrm{~T}^{-1}\right]$ is the exchange

155 rate (a mass fluid flux per unit volume of medium) between the fracture and the matrix 156 continua. 
In (2), the exchange rate is of pseudo steady-state type meaning that the relationship

158 between matrix and fractures depends on pressure gaps between the continua and not on a 159 convolution product of their derivatives with respect to time. In (2), the matrix permeability

$160 \mathbf{k}^{m}$ is assumed small enough to neglect Darcian fluxes in the matrix (compared to that in the

161 fractures) but large enough to enable fluid flux percolation between the matrix and the

162 fractures. Therefore, the matrix permeability is an entry of the exchange rate via the term

$163 \sigma \mathbf{k}^{m}, \boldsymbol{\sigma}\left[\mathrm{L}^{-2}\right]$ being a shape factor tensor that quantifies the mean size of the matrix blocks

164 associated with the fractures included in an elementary volume (for example, the volume

165 corresponding to the elementary meshing employed when solving numerically Eqs (1) and

166 (2)). By construction, the pseudo steady-state assumption in (2) ignores the early transient

167 flow regime between matrix and fractures which may result in erroneous evaluations of

168 exchanged fluid fluxes, especially in the case of weakly permeable matrix media requiring

169 long times for equilibrating their fluid pressure fields with that of fractures (e.g., as in shale

170 gas and shale oil extraction problems). Transient exchange rates between fractures and matrix

171 are the natural outcome of Multiple INteracting Continua (MINC approaches) initially

172 developed in the late eighties (e.g., Pruess and Narasimhan, 1985; Pruess et al., 1990) and

173 more recently reassessed and improved (e.g., Karimi-Fard et al., 2006; Tatomir et al., 2011,

174 de Dreuzy et al., 2013). The MINC models are not incompatible with the notion of mean

175 matrix block size in homogenized fractured systems as each matrix block is viewed as an 176 entity of prescribed size enclosing a nested heterogeneity.

177 Various formulations of the shape factor have been proposed for many modeling 178 applications (Kazemi et al., 1976; Thomas et al., 1983; Coats 1989; Ueda et al., 1989; Lim et 179 al. 1995; Quintard and Whitaker, 1996; Noetinger and Estebenet., 2000) amongst which the 180 formulation proposed by Kazemi et al. (1976) is the one used in this study. This choice is 181 motivated by a quite simple formulation which allows for dealing with diagonal tensors, and 
also introduces the mean matrix block size as a quantity weighting the influence of the matrix

183 permeability tensor to control the fluid fluxes exchanges between matrix and fractures. For

184 diagonal permeability and shape factor tensors, the product $\boldsymbol{\sigma k}^{m}$ is developed as

$185 \quad \boldsymbol{\sigma} \mathbf{k}^{m}=\left(\begin{array}{ccc}k_{x}^{m} / s_{x}^{2} & 0 & 0 \\ 0 & k_{y}^{m} / s_{y}^{2} & 0 \\ 0 & 0 & k_{z}^{m} / s_{z}^{2}\end{array}\right)$

186 with $s_{i}$ [L] $(i=x, y, z)$ the mean matrix block size along the main flow direction $i$. As the

187 exchange rate between the fractures and the matrix is a key feature to the behavior of a dual

188 continuum and some other homogenized approaches (Lemonnier et al., 2010a, b), it makes

189 sense to revisit the item especially regarding the mean matrix block size (which rules the

190 fluxes, provided the fluid pressure fields are correctly calculated).

The Oriented Block Size (OBS) technique that we develop below infers the mean

192 matrix blocks sizes $s_{i} \quad(i=x, y, z)$ from a fractured system by assuming that a rock block

193 enclosing an actual fracture network with various characteristics (e.g., Fig 1) can be turned

194 into a simplified block with an equivalent fracture network composed of three families of 195 planar fractures.

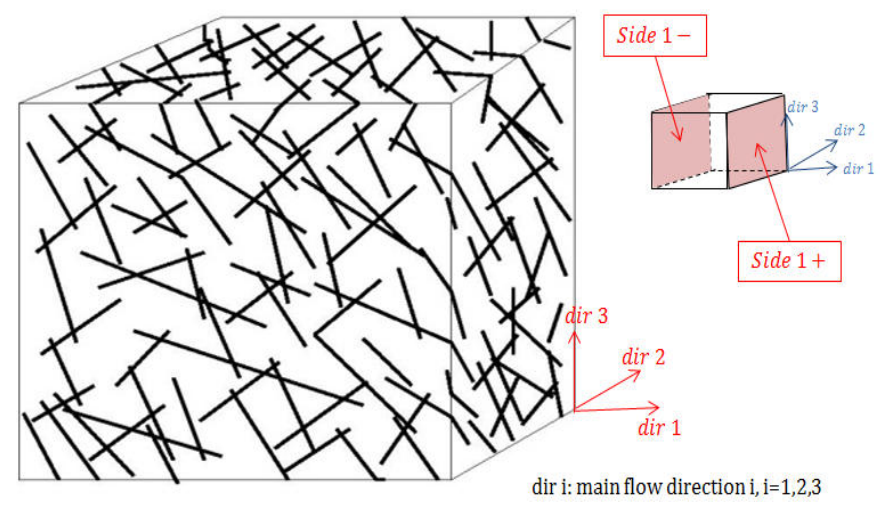

197 Fig. 1. A fractured rock block at the scale of a reservoir grid cell with references to main flow directions and facets of block normal to flow directions. 
200 Each family is defined by a uniform spacing between fractures and a fracture plane normal to 201 one direction of flow (or including the two other directions). This equivalent fracture network (e.g., Fig. 2) which draws the so-called "sugar-cube" configuration as proposed by Warren and Root (1963) and referred to as the WR model hereafter, is conceptually compatible with 204 the notion of mean matrix block size. The three families of WR fractures delimit a 205 parallelepiped elementary block separating neighbor fractures that should coincide with the 206 shape factor as defined in Eq (3). If the whole WR block is wide enough, the three fracture 207 families can be aggregated as a single fracture permeability tensor (or value) and a single 208 fracture porosity for the whole block or its facets. These parameters depend on the size of the 209 elementary matrix block separating the WR fractures. By comparing, or more exactly by 210 identifying permeability and porosity properties of a WR block with that of an actual 211 fractured block, one is able to define the equivalent mean matrix block size of the actual 212 fractured block.
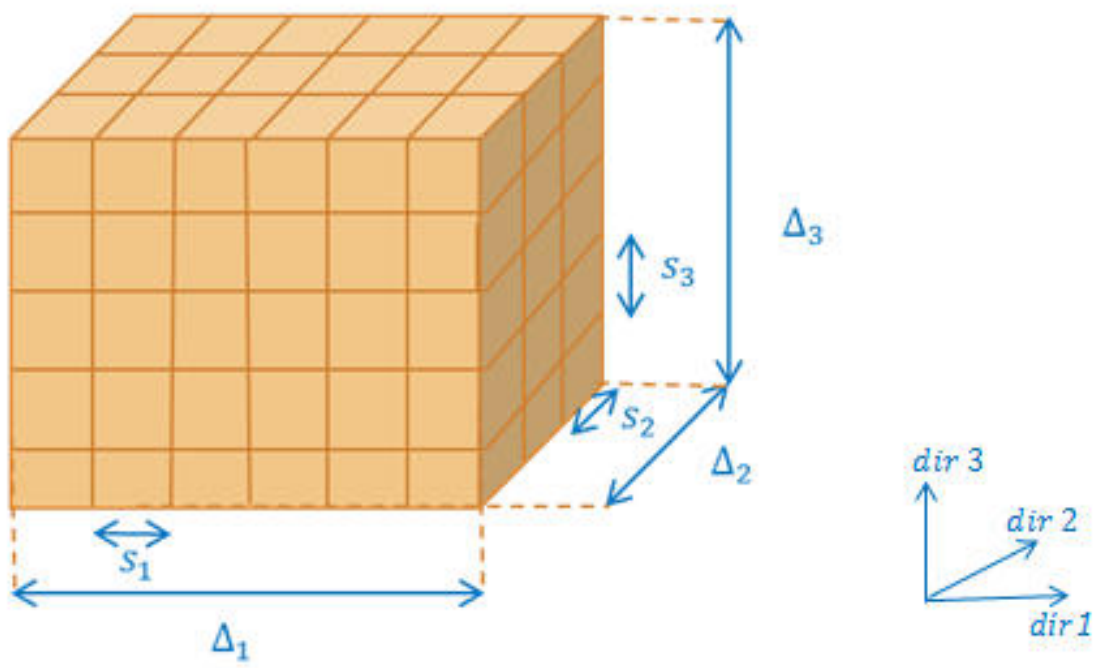

214 Fig. 2. A regular fracture network of three fracture families (a Warren and Root (WR) model) at the scale of a reservoir grid cell with reference to main flow directions, facets of block normal to flow directions, and spacing between fractures. 
Let us take a parallelepiped block housing an actual fracture network as depicted in

220 Fig. 1. The first way to identify a single macroscopic permeability tensor $\mathbf{k}^{f}$ for the block is 221 to employ upscaling approaches, multiple continua theory (Karimi-Fard et al., 2006; Tatomir 222 et al., 2011; Jourdain et al., 2014), analytical solutions (Oda, 1985) or simply to conjecture the 223 entries of the tensor as could be done, for example, in parameterizing a dual porosity approach 224 after having postulated that the approach was convenient for the problem under investigation. A second way is to extract the (diagonal) tensor from the structural properties of the fracture network and its relationships with the homogenization volume (the block) concealing it.

The actual fractured block as depicted in Fig 1 is oriented with its main directions along the main directions of flow indexed by $i=1,2,3$ (here completely equivalent to $i=x, y, z$ for locations in space denoted $\mathbf{x}=(x, y, z)$ but easier to manipulate when incrementing the 230 index). The block size in direction i is denoted $\Delta_{i}$ and the sides delimiting the block are also 231 indexed by i but for limits normal to the main direction i. In addition, block sides are labelled $i-$ or $i+$ according to their respective location upstream or downstream along direction i.

233 Assuming that the fractured block is well connected, the mean permeability of the block along 234 a direction $\mathrm{i}$ can be calculated as the average over the sides $i$ - and $i+$ of the local 235 permeability of fractures intercepting the sides. This yields

$$
k_{i}^{F N-S}=\frac{1}{2\left(\Delta_{i+1} \Delta_{i+2}\right)}\left(\sum_{n=1}^{N f_{i-}} k_{n} l_{n} e_{n}+\sum_{n=1}^{N f_{i+}} k_{n} l_{n} e_{n}\right)
$$

237 In the above equation, $i$ is a cycling index such that, e.g., $i+1=3$ when $i=2$ and $i+1$ returns 238 to 1 when $i=3 . k_{i}^{F N-S}\left[\mathrm{~L}^{2}\right]$ is the macroscopic fracture permeability of the fractured block 239 along direction $i, \Delta_{i+1} \Delta_{i+2}\left[\mathrm{~L}^{2}\right]$ is the total surface area of sides $i-$ and $i+$ these being 240 intercepted by a number of fractures $N f_{i-}$ and $N f_{i+} \cdot k\left[\mathrm{~L}^{2}\right]$ is the local permeability of a 
241 fracture seen as intercepting the side of the block over an apparent length $l$ and with apparent

242 fracture aperture $e[\mathrm{~L}]$.

By re-using the same notations for directions and sides in a rock block modeled as a

244 WR network (Fig. 2), one can also calculate the entries $k_{i}^{W R}$ of the diagonal fracture 245 permeability tensor of the WR block along directions i. The three fracture families of the WR 246 network are also indexed by $\mathrm{i}$ with the same notation as for the block sides, i.e., a fracture 247 family i corresponds to fracture planes normal to direction i. A family $\mathrm{i}$ is of uniform spacing $248 s_{i}[\mathrm{~L}]\left(s_{i}\right.$ is measured along direction i, see Fig. 2), counts $N f_{i}$ fractures with a uniform 249 scalar permeability $k_{i}$ and a uniform fracture aperture $e_{i}$. With these settings and the 250 assumption that flow only occurs in the fractures, the total flow rate entering or exiting the 251 WR fractured block through a side $i$ normal to the direction $i$ can be expressed as

$Q_{i}=-\nabla_{i} P\left(\sum_{j \neq i} N f_{j} e_{j} \Delta_{k ; k \neq i, k \neq j} \frac{k_{j}}{\mu}\right)=-\nabla_{i} P \frac{k_{i}^{W R}}{\mu} \Delta_{i+1} \Delta_{i+2}$

253 For the sake of simplicity, the gravity components of flow have not been accounted for in (5).

$254 N f_{j} e_{j} \Delta_{k ; k \neq i, k \neq j}$ represents the total surface of flow developed by the family fracture $j$ through 255 the side $i$ of the block, $\Delta_{i+1} \Delta_{i+2}$ is the total surface area of the side $i$, and $k_{i}^{W R}$ is the 256 macroscopic fracture permeability of the WR block along direction $i$. The equality in (5) 257 comes down to a direct identification of the three terms of the macroscopic permeability $\mathbf{k}^{W R}$ 258 as

$259\left[\begin{array}{c}k_{1}^{W R} \\ k_{2}^{W R} \\ k_{3}^{W R}\end{array}\right]=\left[\begin{array}{ccc}0 & 1 / \Delta_{2} & 1 / \Delta_{3} \\ 1 / \Delta_{1} & 0 & 1 / \Delta_{3} \\ 1 / \Delta_{1} & 1 / \Delta_{2} & 0\end{array}\right] \cdot\left[\begin{array}{c}N f_{1} e_{1} k_{1} \\ N f_{2} e_{2} k_{2} \\ N f_{3} e_{3} k_{3}\end{array}\right]$

260 In the context of scaling the values $k_{i}^{W R}$ so they become equivalent to calculated values in a 261 rock block encapsulating an actual fracture network, the number $N f_{i}$ of WR fractures, their 
aperture $e_{i}$ and their permeability $k_{i}$ become the unknowns of the problem. Therefore, it

263 makes sense to invert the linear system of equation (6) which yields

$264 \quad N f_{i} e_{i} k_{i}=\frac{\Delta_{i}}{2} \sum_{j=1}^{3}(-1)^{\delta_{i, j}} k_{j}^{W R}$

265 with $\delta_{i, j}$ the Kronecker delta function, $\delta_{i, j}=1$ if $i=j$ and $\delta_{i, j}=0$ if $i \neq j$. By considering the structure of a WR fracture network, one can write

$$
N f_{i}\left(e_{i}+s_{i}\right)=\Delta_{i} \Rightarrow \frac{N f_{i} e_{i}}{\Delta_{i}}=\frac{e_{i}}{e_{i}+s_{i}}
$$

268 Note in the above expression that counting $N f_{i}$ fractures assumes the presence of $N f_{i}-1$

269 fractures inside the block and that the two sides $i$ of the block are each bounded by half a 270 fracture of family $i$ with half the aperture $e_{i}$ counted in the block. Introducing (8) in (7)

271 results in

$272 \quad k_{i}=\frac{1}{2}\left(1+\frac{s_{i}}{e_{i}}\right) \sum_{j=1}^{3}(-1)^{\delta_{i, j}} k_{j}^{W R}$

273 The expression (9) will be used later for the purpose of identification between an actual 274 fractured block and a WR block. Let us look at the porosity properties of the WR block. The fracture porosity $\phi^{W R}[-]$ of 276 the whole WR block and the fracture density $\phi_{i}^{W R-S}[-]$ at a side $i$ defined as the porosity of 277 fracture network at a side of the block (the ratio of the surface area of open fractures at a side 278 to the total surface of the side) can also be derived as

$279 \quad \phi^{W R} \approx \sum_{i=1}^{3} \frac{N f_{i} e_{i} \Delta_{i+1} \Delta_{i+2}}{\Delta_{1} \Delta_{2} \Delta_{3}}=\sum_{i=1}^{3} \frac{N f_{i} e_{i}}{\Delta_{i}}$

$$
\phi_{i}^{W R-S} \approx \sum_{l \neq i} \frac{N f_{l} e_{l}}{\Delta_{l}}
$$


Notably, the expressions in (10) and (11) are rather simple but are approximations since the

282 intersections of fractures are counted twice in the porosity values. This was found of 283 negligible influence for classical block sizes and fracture apertures. Subtracting (11) from (10)

284 returns the term $N f_{i} e_{i} / \Delta_{i}$ which also appears in Eq (8). Therefore, another way to express the 285 relationship between the local WR fracture permeability $k_{i}$ and the macroscopic permeability $286 \mathbf{k}^{W R}$ in (9) is

$$
k_{i}=\frac{1}{2\left(\phi^{W R}-\phi_{i}^{W R-S}\right)} \sum_{j=1}^{3}(-1)^{\delta_{i, j}} k_{j}^{W R}
$$

288 Both expressions (9) and (12) are employed to define the matrix block size $s_{i}$ (in 9).

If the WR network is equivalent regarding its hydraulic properties to the actual 290 fracture network, it is expected that $\phi^{W R}, \phi_{i}^{W R-S}$, and $k_{j}^{W R}$ are similar to the equivalent 291 properties in the actual block of fracture network, respectively denoted as $\phi^{F N}, \phi_{i}^{F N-S}$, and $292 k_{j}^{F N-S}$ (see (4) for the latter term). It is also expected that the WR network, while being still 293 equivalent to the actual fractured block, can inherit some properties (parameters) of a 294 homogenized model such as the mean matrix block sizes of the medium and the permeability 295 tensor at the macroscopic scale of a fractured block. By imposing these properties in (9) and 296 (12), and after a few algebraic manipulations (see Appendix A for details), an expression of 297 the mean matrix block sizes in a homogenized fractured block can be written as

$$
s_{i} \approx \frac{\sum_{j=1}^{3}(-1)^{\delta_{i, j}} k_{j}^{F N-S}}{\left(\phi^{F N^{*}}-\phi_{i}^{F N-S^{*}}\right) \sum_{j=1}^{3}(-1)^{\delta_{i, j}} k_{j}^{f}}
$$

$299 k_{i}^{F N-S}(i=1,2,3)$ are the permeability values at the sides $i$ of the actual fractured block, $300 k_{i}^{f}(i=1,2,3)$ are the entries of the diagonal permeability tensor of an homogenized medium 301 equivalent to the fractured block (e.g., that of a dual porosity model), and $\phi^{F N^{*}}$, 
$\phi_{i}^{F N-S^{*}}(i=1,2,3)$ are rescaled block and side porosities of the actual fractured block. These

303 rescaled porosities of dimension $\left[\mathrm{L}^{-1}\right]$ (a porosity per unit fracture aperture) are calculated 304 over the skeleton of the actual fracture network to which each fracture is assigned a unit 305 fracture aperture.

In addition to postulating the equivalence between a WR block and the actual fractured block, the assumptions allowing us to derive (13) are twofold. First, the actual fracture 308 network is a good candidate for homogenization with the meaning that there exist macroscopic properties as mean matrix block size and diagonal permeability tensor characterizing the hydraulic behavior of the network at the large scale (at least, the scale of a mesh of a homogenized model). Second, a WR network exists (as that investigated by way of equations 5 to 12) but with uniform fracture aperture $e_{f}$ over its three fracture families and

313 still equivalent to the actual fracture network (see Appendix A for details). There is no clear 314 criterion (except dealing with a dense and well-connected network) allowing us to state 315 beforehand whether or not a given fracture network would follow the above assumptions.

316 Eventual criteria would also depend on the flow processes and mechanisms targeted for 317 further applications at the large scale. It is worth to note that Eq. (13) depends on both the facet permeability values of the 319 actual fractured block $k_{j}^{F N-S}$, and the structural properties of the actual fracture network 320 skeleton in the form of porosities $\phi^{F N^{*}}$ and $\phi_{i}^{F N-S^{*}}$. These features make that applicability of 321 (13) is conditioned by a good knowledge of the actual fracture network geometry and, as a 322 downside, renders the method hardly applicable to poorly-known natural systems. In the end, 323 Eq. (13) should be mainly used in problems dealing with homogenization of systems with 324 well-known geometry and discretization of synthetic fracture networks (as done for instance 325 in reservoir engineering when passing from a geological model to a tractable flow model). 
This notwithstanding, the OBS technique can also deliver another form of the mean matrix block size. By manipulating the expression of the side block permeability of the actual fractured block in (4), scaling the subsequent expression with the side block porosities $\phi_{i}^{F N-S}$ and making use of (13) (details are provided in Appendix A), another form of the mean matrix

330 block size comes up as

$331 s_{i} \approx \frac{2 e_{f} \bar{k}}{\sum_{j=1}^{3}(-1)^{\delta_{i, j}} k_{j}^{f}}$

332 This form introduces the existence of a mean single fracture aperture $e_{f}[\mathrm{~L}]$ (which is also the 333 uniform aperture mentioned above for the WR network) and a mean single-fracture 334 permeability $\bar{k}\left[\mathrm{~L}^{2}\right]$ at the scale of the whole actual fractured block. These two quantities are 335 additional assumptions to that discussed regarding (13) for the applicability of (14).

336 Even though these assumptions may appear very restrictive, they give the possibility 337 to infer mean matrix block sizes from poorly known and hardly accessible fracture networks 338 as often encountered in field case applications. The entries $k_{j}^{f}$ of the permeability tensor of 339 the whole fractured block can be evaluated by way of hydraulic tests; preferably interference 340 testing between distant wells that avoid bias stemming from an environment close to the 341 tested well that would not be representative of the fracture network at a larger scale. Values of 342 uniform single-fracture aperture $e_{f}$ and uniform single-fracture permeability $\bar{k}$ are harder to 343 infer because data obtained for instance from optic imaging of boreholes (for $e_{f}$ ) and flow or 344 production logs (for $\bar{k}$ ) may reveal not representative of the whole network. It remains that 345 the matrix block size calculation in (14) is feasible without resorting to any knowledge on the 346 structure of the actual fracture network. It is obvious that the subsequent inferred value of 347 mean matrix block size should be taken as an order of magnitude (then refined for instance by 348 model inversion) instead of a robust pinpoint value. 
In the following comparing: 1- the OBS technique with other geometrical techniques,

and 2- the dual porosity approach (handling matrix block sizes $s_{i}$ ) with finely discretized

351 networks, we address the relevance of the simplified expression in (14) under the assumption

352 that the skeleton of the fracture network is known (as is the case with other geometrical

353 methods). We prescribe to each fracture a uniform aperture and a uniform fracture

354 permeability. The skeleton is then discretized and the entries $k_{j}^{f}$ of the permeability tensor are

355 calculated by performing numerical "permeameter" experiences (i.e., calculating fluid fluxes

356 between opposite facets of the fractured block under prescribed Dirichlet boundary conditions

357 while the other facets of the block are of no-flow type).

\section{Comparison with structural-geometrical approaches}

As shown from a theoretical standpoint, the OBS technique defines a mean matrix

block size as a measure drawn from geometrical and structural properties of a discrete fracture network and its equivalent representation via a regular "sugar cube" network. In theory, no reference to any calculation of flow at the large scale is evoked in obtaining the OBS matrix block size, which renders the technique comparable in its spirit to other previous approaches also based on geometrical-structural characteristics of the discrete fracture network.

In the following, the OBS evaluation of matrix block sizes is compared with three other types of geometrical calculations, namely: the geometrical imbibition (GI) technique (Bourbiaux, 1997), the extended geometrical imbibition (EGI) technique (Bourbiaux et al., 2006), and the mean spacing (MS) technique (Narr, 1993). The main backgrounds of GI, EGI, and MS are summarized (sometimes slightly enhanced, as for EGI) and presented with

371 notations consistent with that of the present work in Appendix B. GI and EGI techniques are 372 only applicable (in their original version) to two-dimensional fracture networks and model the 373 distance between any location in the matrix and the nearest fracture of the DFN. MS is 
available for two- and three-dimensional systems and infers the mean lag distance between

375 two neighbor fractures along the main directions of flow in a fractured block. All the 376 geometrical methods need the detailed geometry of the DFN, although OBS could be used 377 without it (See Section 2). But for a fair comparison we assume for all methods that the 378 skeleton of the fracture network is known.

The comparison of OBS, GI, EGI, and MS is conducted for the two horizontal 380 directions of a three-dimensional fractured block (100 $\mathrm{m}$ on a side) consisting of two families 381 of near-vertical fracture planes. In the first test, a fracture family denoted $A$, is oriented with 382 an azimuth of $100^{\circ}$ counted positive anticlockwise from the main direction $x$ of the fractured 383 block. The second fracture family denoted $B$ is oriented $10^{\circ}$. The spacing between fractures of 384 family $B$ is kept constant at $7 \mathrm{~m}$, as the spacing of family $A$ is varied between 2 and $8 \mathrm{~m}$ for 385 different realizations of the DFN (two examples reported in Fig. 3).

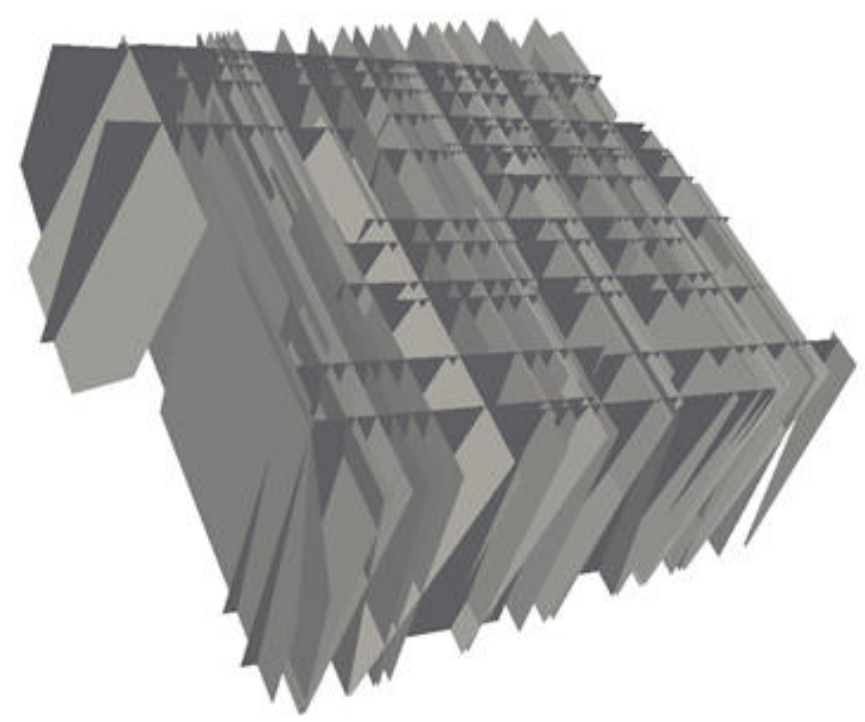

a families $A$ and $B$ with mean spacing of $7 \mathrm{~m}$.

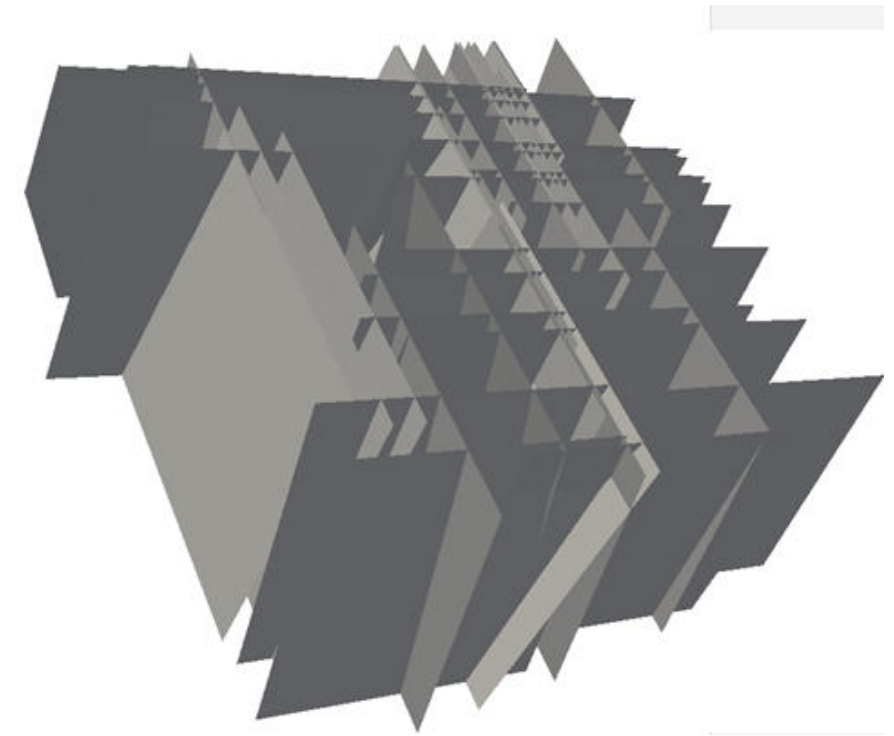

b

Fig. 3. Examples of random discrete fracture networks (DFN) with two near-vertical fracture families at the scale of a reservoir grid cell. The azimuths of family $A$ and $B$ are $100^{\circ}$ and $10^{\circ}$, respectively. DFN a: family $A$ (resp. $B$ ) with mean spacing of $2 \mathrm{~m}$ (resp. $7 \mathrm{~m}$ ); DFN b: 
If we denote as $s_{x}$ and $s_{y}$ the mean matrix block sizes along the $x$ and $y$ horizontal directions

392 of the fractured block, in view of the orientations of fracture families $A$ and $B, s_{x}$ should be 393 close to the mean spacing of $A$ (i.e., $2-8 \mathrm{~m}$ ), and $s_{y}$ close to the spacing of $B$ (i.e., $7 \mathrm{~m}$ ). Fig. 3944 reports on sought values of $s_{x}$ and $s_{y}$ for different methods of calculation with specifically 395 the EGI technique rendering two sets of measures (see Appendix B) - small-EGI, large-EGI 396 as the technique assumes the existence of two types of matrix block interacting with the 397 fracture network during flow.
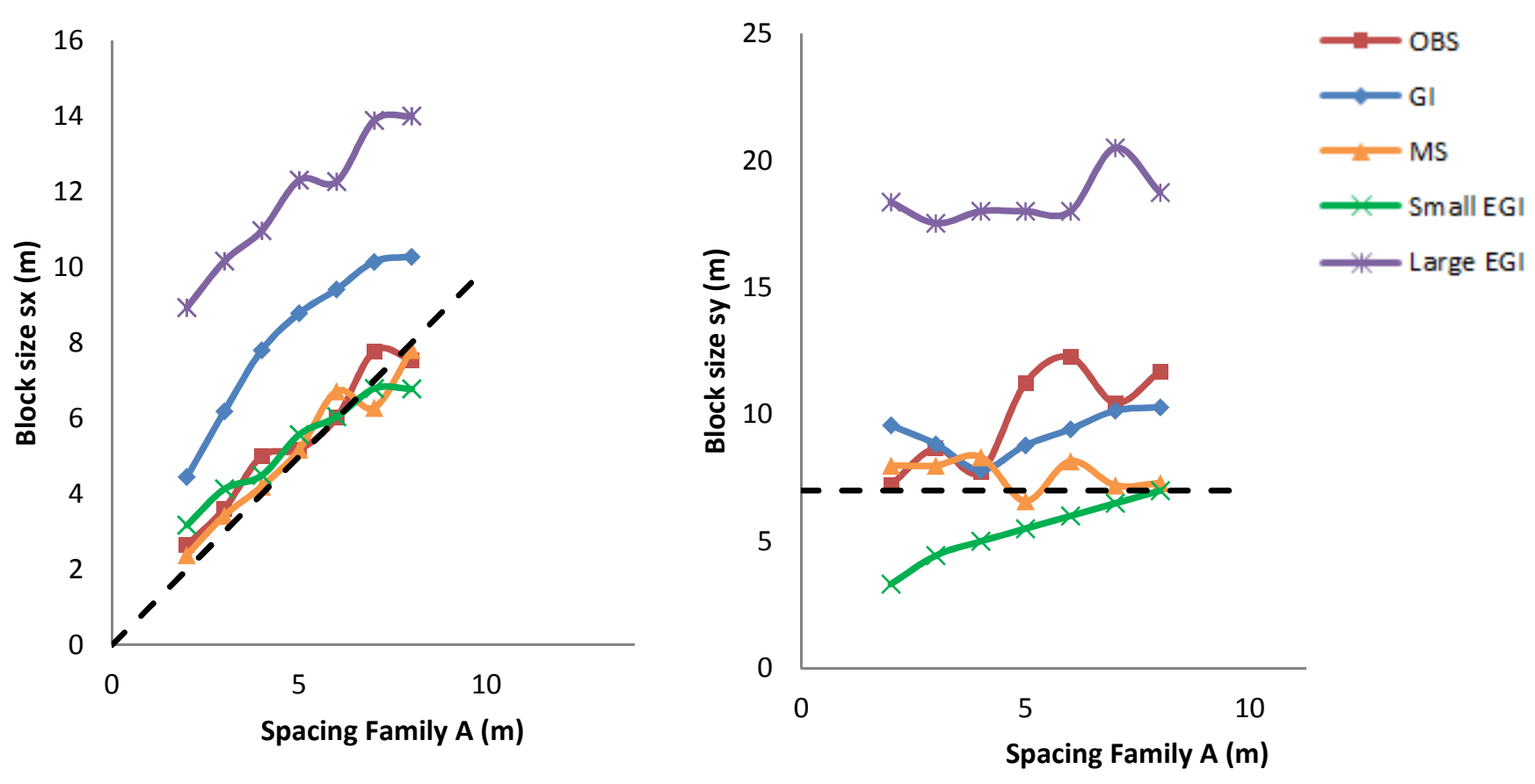

a

398 Fig. 4. Mean matrix block sizes $s_{x}$ and $s_{y}$ as functions of the spacing of fracture family $A$ 399 (fracture networks in Fig. 4) for different methods of calculation. OBS = oriented block size 400 method, GI = general imbibition method, EGI = enhanced general imbibition method (with 401 "small" and "large" sizes of matrix blocks), and MS = mean spacing method.

402 In general, the OBS calculations retrieve the expected values of $s_{x} \approx 2-7 \mathrm{~m}$ as a 404 function of the spacing of fracture family $A$ (Fig. 4a). The size $s_{y}$ which should be constant at 
$7 \mathrm{~m}$, actually evolves with the spacing of family $A$ and is overestimated of $10 \%$ to $50 \%$ (Fig.

406 4b). This overestimation cannot be the consequence of an actual fracture network that would 407 be far from a regular WR representation since the actual network is simple and made of two 408 perpendicular fracture families with directions almost parallel to the $x$ and $y$ directions of the 409 fractured block. Nevertheless, we noted that increasing the spacing of the fracture family $A$ 410 also diminished the connectivity of the DFN with a few subdomains almost free of any 411 fracture and poorly connected to the facets of the fractured block. It is noteworthy that 412 estimates of effective properties of the DFN, especially porosities (or their influence on 413 macroscopic permeabilities in Eq. (15)), both at the facets and inside the block are key 414 features to the OBS calculations (see Section 2). Since less connected networks return weaker 415 porosity values, the equivalent WR network assigned with those porosities will contain less 416 fractures and result in increased matrix block sizes extracted from the equivalence between 417 the WR network and the DFN. Compared with the expected values, matrix block sizes $s_{x}$ and $s_{y}$ extracted from the

419 GI technique tend to be overestimated. This result is foreseeable because GI usually 420 experiences some difficulties when dealing with DFN encompassing both small and large 421 matrix blocks. These difficulties are the consequence of the oversimplified fitting with a 422 second degree polynomial of the so-called invasion area curve calculated by the method as the surface area in the matrix domain located at a given distance from the closest fracture of the

424 system (see Appendix $B$ ). Regarding EGI, the "small block" estimates $s_{x}$ are in the correct 425 range 2-8 $\mathrm{m}$ when the size $s_{y}$ is always overestimated. For their part, the "large block" 426 estimates in EGI are always more than twice the expected values. Finally, the MS method 427 infers correct values of $s_{y}$ and $s_{y}$ whichever the investigated DFN and the spacing of fracture 428 families $A$ and $B$. Notably, the MS method is weakly influenced by the fracture network 429 connectivity which might become a drawback when dealing with sparse and poorly connected 
fracture networks. In that case MS will still measure the mean lag distance separating two neighbor fractures, as a poorly connected network tends to conceal a few cluster of large matrix blocks in the system. In that case mean matrix block sizes from MS would be underestimated.

In the OBS technique, whose specificity is seeking the equivalence between the actual

435 DFN and a regular WR network, this equivalence seems intuitively easier to achieve for 436 DFNs with fracture families whose principal orientations are close to the main directions of 437 the whole block. Therefore, it makes sense to address the capabilities of the method under less 438 favorable conditions where actual fractures do not line up with the main block directions. We 439 re-handled the comparison of matrix block sizes drawn from fracture networks still made of 440 two almost vertical fracture families, but this time with a constant spacing of $3 \mathrm{~m}$ for family $441 A, 5 \mathrm{~m}$ for family $B$, and varying the orientation of the families with respect to the main 442 directions $x$ and $y$ of the block. The fracture family $A$ is still oriented $100^{\circ}$ (counted positive 443 anticlockwise) with respect to the $x$ direction and the orientation of family $B$ is varied between 4440 and $70^{\circ}$ with respect to $x$ (Fig. 5). In view of the geometrical settings of the DFNs, the 445 matrix block size $s_{x}$ should be close to $3 \mathrm{~m}$ and $s_{y}$ close to $5 \mathrm{~m}$ when the fracture family $B$ is 446 almost orthogonal to family $A$ (azimuth of $B=0-10^{\circ}$ ). Block sizes $s_{x}$ should then slightly 447 decrease as $s_{y}$ should increase when the direction of fracture family $B$ departs from 448 orthogonality with $A$.

449 The GI method systematically overestimates both $s_{x}$ and $s_{y}$ in each configuration of 450 the fracture network. The EGI technique still tends to overestimate $s_{x}$ and $s_{y}$ with its "large 451 block" measure while correct or slightly underestimated values are found with the "small 452 block" measure. In any case, both GI and EGI are weakly sensitive to the fracture family 453 orientations with almost constant values $s_{x}$ and $s_{y}$ irrespective of the azimuth prescribed to 
454 fracture family $B$ in the DFN (Fig. 6). This result is consistent with the fact that both 455 techniques model the surface occupied by matrix domains in the fractured block as a function 456 of the distance to the nearest fracture (Appendix B). This measure reveals far less sensitive to 457 fracture orientations than to fracture spacing.

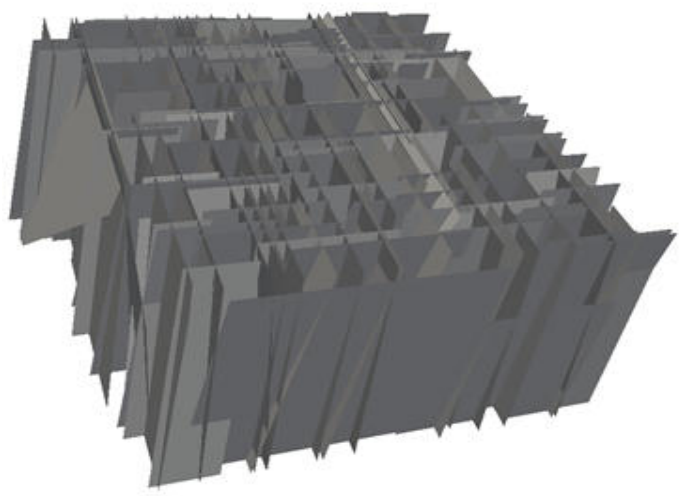

a

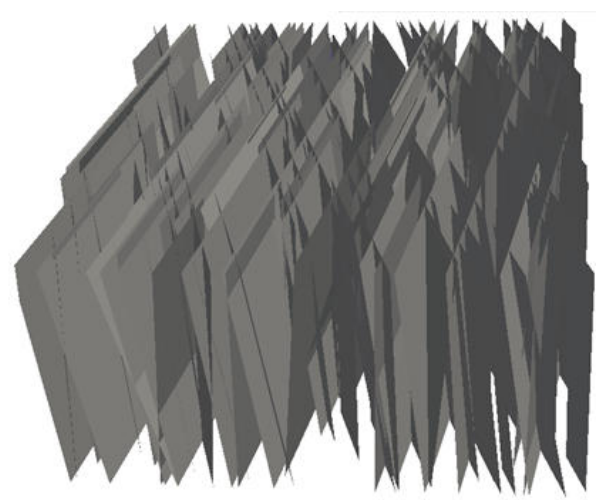

b

458 Fig. 5. Examples of random discrete fracture networks (DFN) with two near-vertical fracture families at the scale of a reservoir grid cell. The mean spacing of fracture families $A$ and $B$ are prescribed at $3 \mathrm{~m}$ and $5 \mathrm{~m}$, 460 respectively, while the azimuth of family $A$ is kept at $100^{\circ}$ and the azimuth of family $B$ is varied between $0^{\circ}$ $461 \quad\left(\mathrm{DFN}\right.$ a) and $70^{\circ}(\mathrm{DFN}$ b).
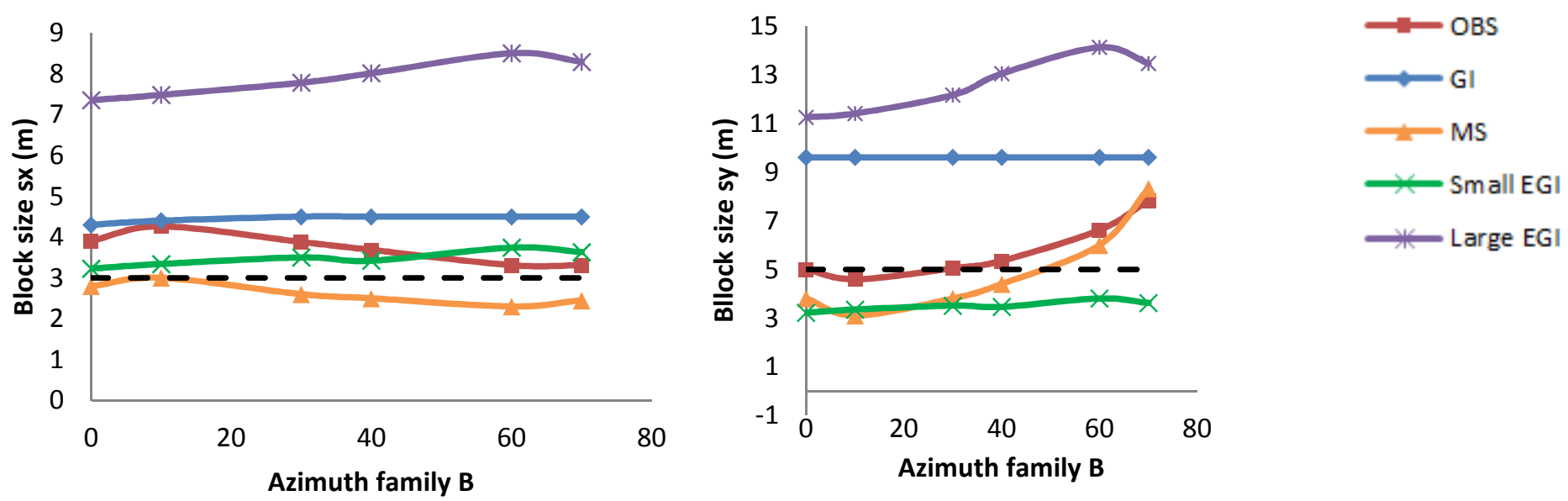

a

b

463 Fig. 6. Mean matrix block sizes $s_{x}$ and $s_{y}$ as functions of the azimuth of fracture family $B$ (fracture networks

464 in Fig. 5) for different methods of calculation. OBS = oriented block size method, GI = general imbibition

465 method, EGI = enhanced general imbibition method (with "small" and "large" sizes of matrix blocks), and MS =

466 mean spacing method. 
The MS and OBS techniques infer very similar matrix block size values, these being

468 sometimes slightly underestimated by MS and slightly overestimated by OBS. For azimuths

469 of the fracture family $B$ between 0 and $45^{\circ}$, the estimated $s_{x}$ with both MS and OBS are close

470 to the expected value of $3 \mathrm{~m}$ and stay almost constant whichever the orientation of family $B$.

471 Concerning $s_{y}$, the expected value of $5 \mathrm{~m}$ is retrieved by OBS and underestimated at 3-4 $\mathrm{m}$

472 by MS. For azimuths of the fracture family $B$ between 45 and $70^{\circ}$, both methods return, as

473 expected, $s_{x}$ values that slightly decrease, as $s_{y}$ values increase from approximately $5 \mathrm{~m}$ up

474 to $8 \mathrm{~m}$. OBS mainly captures the projection of the fracture planes onto the facets delimiting

475 the fractured block (see Section 2 and Appendix A), which is obviously sensitive to fracture

476 orientations. In the same vein, MS evaluates the mean distance between fractures along the

477 main directions of the fractured block with the obvious consequence of increasing the

478 apparent distance when fracture planes are not normal to the direction of measure.

479 Nevertheless, both methods provide valuable results for dense fracture networks or fractured

480 blocks wide enough to enclose a large number of fractures allowing for significant statistical

481 measures of fracture spacing (MS) or block-side and inner-block hydraulic properties (OBS).

Notwithstanding other considerations such as computation times (see hereafter), OBS

and MS techniques seem to outperform GI and EGI in extracting mean matrix block sizes

from fractured system. We noted however that OBS is sensitive to the loss of connectivity in a

485 fracture network with the consequence of increasing the inferred matrix block size. This

486 artificial increase might result in biased evaluations of fluid flux exchanges between fracture

487 and matrix media. Numerical exercises comparing discrete fracture network outputs and their

488 dual porosity representation with OBS-sized matrix blocks are conducted to answer this

489 question. The other geometrical techniques GI, EGI, and MS are also tested. We remind that

490 these three numerical techniques are in essence only applicable when a prior knowledge of the

491 fracture network geometry is available, while the OBS technique might by applied either on 
492 known or unknown geometries (see Section 2). For a fair comparison of all techniques

493 hereafter, we consider that the fracture network geometry is known.

494

495

\section{Two-dimensional numerical test cases}

As already mentioned, dual continua representations of discrete fracture networks are conducive to drastic reductions in computation costs but require carefully designed settings to adequately represent both conductive and capacitive properties of a fractured porous medium subject to Darcian flow. We address here two phase flow in both DFN and dual porosity models. The setup of calculations is dimensioned to represent large laboratory analogs of flow in fractured media as conducted for instance in "Hele-Shaw" cells (e.g., Park and Homsy, 1984; Folch et al., 1999). We remind that we are interested in the assessment of mean matrix block size from different geometrical-structural techniques that always manipulate relative quantities as the spacing of fractures compared to the block size, or fracture traces intercepted by block facets. Therefore, our findings from numerical experiments at the scale of a lab device should not be hampered by loss of generality. In addition, we perform calculations, especially in the context of DFN discretization, over synthetic fracture networks

508 with regular fracture orientations. This choice reduces discretization efforts but is mainly employed herein because it ensures accuracy of reference calculations in a DFN compared with that from a dual porosity model. Even though sophisticated meshing techniques and advanced numerical methods exist, it was found that thin fracture elements in unstructured

512 meshing tend to smear the calculation of their state variables over the large matrix blocks.

513 This feature is not suited to compare (local) DFN and (large scale) dual porosity calculations 514 of diffusive flow. systems (of unit thickness) that only neglect gravity-driven flow. Notably, the various 
techniques employed in this study to calculate mean matrix block sizes are not sensitive to

518 gravity-driven flow and only manipulate geometrical considerations on the fracture network or equivalences in permeability-porosity between an actual fractured block and a sugar-cube model. Two-phase flow in a DFN is performed over a fractured system of $3 \mathrm{~m}$ length and 1.5 521 m width finely discretized by 11590 square elements for an accurate representation of both 522 the fracture network and matrix. The system is also roughly discretized by only 920 square 523 elements of a dual-porosity, single-permeability model with matrix block sizes extracted from 524 the DFN via the EI, EGI, MS and OBS techniques (see Section 3). Two-phase flow is 525 numerically solved by means of a finite volume technique and uses an implicit-in-time 526 scheme for time integration of the pressure equation while an explicit-in-time scheme is used 527 for time integration of either the water or oil mass balance. To avoid unfair comparisons 528 between GI, EGI, MS, and OBS, a simple fracture network is delineated with fractures only 529 parallel to the main flow directions $x$ and $y$ of the system. Dead ends of the fracture network 530 are also removed since in essence they are always accounted for in the fracture-matrix 531 relationship by GI and EGI methods when MS and OBS might not see these dead-ends 532 because they are not counted in MS or do not participate to side-block properties in OBS.

533 The first fractured system investigated (Fig. 7) is initially saturated with oil and 534 percolated by water injected from the western boundary taken as a Neumann condition 535 prescribing a constant-in-time water flux. The eastern boundary of the system is of Dirichlet 536 type while North and South boundaries are of no-flow type. Table 1 indicates the local 537 hydraulic properties of each medium (fractures, matrix) in the DFN, Table 2 reports on inner538 block and block-side properties used by the OBS method to calculate matrix block sizes, and 539 Table 3 gathers the various matrix block sizes $s_{x}$ and $s_{y}$ obtained from the GI, EGI, MS and 540 OBS methods. 


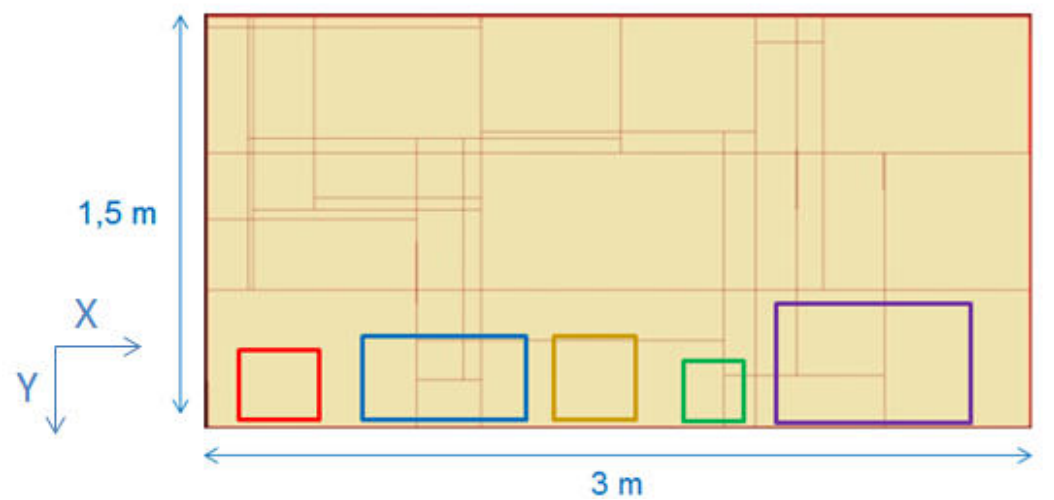

$3 \mathrm{~m}$

543 Fig. 7. Two-dimensional fracture network serving as a system finely discretized or handled as a dual-porosity 544 model for the purpose of flow dynamics comparison. The size of matrix blocks in a dual porosity approach are 545 reported as colored frames, from left to right: Red = oriented block size method, Blue = general imbibition 546 method, Orange = mean spacing imbibition method, Green and Purple $=$ small and large sizes from enhanced 547 general imbibition method.

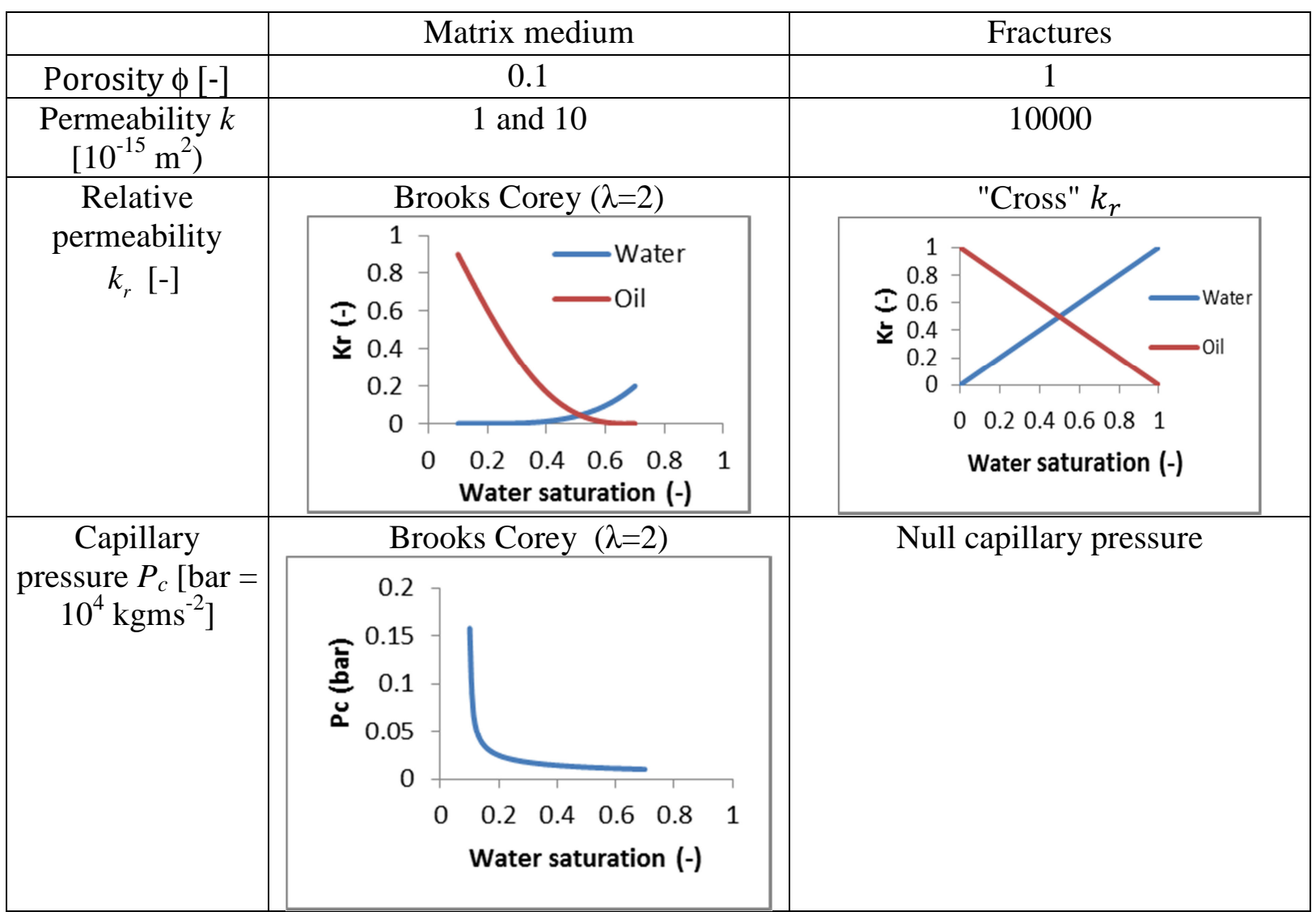

5507 and 10 . The relative permeability and capillary pressure as functions of water saturation in the matrix obey the

551 Brooks and Corey model (1964) with $\lambda(=2)$ the so-called pore-size distribution index. 
553 Table 2. Main macroscopic parameters of the fractured block in Fig. 7 to infer via the oriented block size

554 technique the mean matrix block size of a dual porosity model. $k_{f}, \phi_{f}$ respectively are the permeability and

555 porosity of the whole block, $k^{F N-S}, \phi^{F N-S}$ respectively are the permeability and porosity of the fracture 556 network at the sides (normal to $x$ and $y$ directions) of the block.

\begin{tabular}{|c|c|c|c|c|c|}
\hline Block sizes & OBS & GI & MS & $\begin{array}{c}\text { EGI- } \\
\text { large }\end{array}$ & $\begin{array}{c}\text { EGI- } \\
\text { small }\end{array}$ \\
\hline$s_{x}[\mathrm{~m}]$ & 0.360 & 0.6 & 0.35 & 0.696 & 0.257 \\
\hline$s_{y}[\mathrm{~m}]$ & 0.306 & 0.3 & 0.345 & 0.494 & 0.257 \\
\hline
\end{tabular}

5597.

Two different types of flow are simulated, the first one with low water injection rate of $0.1 \mathrm{~m} /$ day in the fractures and low matrix permeability of $10^{-15} \mathrm{~m}^{2}$, the second one with higher 563 injection rate of $1 \mathrm{~m} /$ day and higher matrix permeability of $10^{-14} \mathrm{~m}^{2}$. On the one hand, the 564 first scenario with small water fluxes in the fractures and weakly permeable matrix enhances 565 capillary effects as the origin of pressure gradients between fracture and matrix and 566 subsequent exchange rates between both media Due to capillary effects in the matrix and 567 absence of these in the fractures, the oil pressure in the matrix is higher than that in the 568 fractures and oil is ejected from the matrix (or water invades the matrix). On the other hand, 569 the second scenario with high injection velocities favors "piston" flow in the fractures and 570 enhances fracture-matrix exchanges as the consequence of the excess of water pressure in 571 fractures compared with oil pressure in the matrix. Water invades the matrix and the process 
572 is enhanced by the contrast of mobility (the ratio $k r / \mu$ ) between oil and water phases which

573 triggers rapid water invasion along the fractures and early leaching of matrix blocks.

574 To reinforce these assertions about flow scenarios with contrast between capillary and

575 viscous forces to extract oil from matrix blocks, we also calculated a dimensionless capillary

576 number based on the evaluation of water fluxes invading matrix blocks versus expulsion of oil

577 from the matrix to fractures by capillary pressure contrasts. With steady-state flow sweeping

578 oil from the system by forced water injection at one side of the fractured block, the mean

579 water pressure gradient in the system is evaluated as

$580 \quad\left|\nabla P_{w}\right| \approx \frac{V_{i n j} \mu_{w}}{k_{i}^{f}}$

$581 P_{w}\left[\mathrm{ML}^{-1} \mathrm{~T}^{-1}\right]$ is the water pressure in both the fractures and the matrix, $V_{i n j}\left[\mathrm{LT}^{-1}\right]$ is the 582 injection velocity of water at the upstream side of the fractured block, $\mu_{w}\left[\mathrm{ML}^{-1} \mathrm{~T}^{-1}\right]$ is the 583 dynamic viscosity of water, and $k_{i}^{f}\left[\mathrm{~L}^{2}\right]$ is the equivalent fracture permeability of the whole 584 block along the direction i of water injection. Regarding the capillary pressure gradient, we 585 assume a null capillary pressure in the fractures (open medium of unit porosity) and we take 586 in the matrix the maximal capillary pressure $P_{c}^{\max }$ given by relationships capillary pressure 587 saturation (see, e.g., Table 1). The capillary pressure gradient between matrix and fractures is 588 then approximated as

$589 \quad\left|\nabla P_{c}\right| \approx \frac{P_{c}^{\max }}{S_{\min } / 2}$

590 with $s_{\min }[\mathrm{L}]$ the smallest dimension (in either directions $\mathrm{x}$, or $\mathrm{y}$ or $\mathrm{z}$ ) of the mean matrix

591 block size. A dimensionless capillary number balancing capillary gradient with water pressure

592 gradient can be expressed as

$593 \quad n_{c}=\frac{\left|\nabla P_{c}\right|}{\left|\nabla P_{w}\right|} \approx \frac{2 P_{c}^{\max } k_{i}^{f}}{S_{\text {min }} \mu_{w} V_{i n j}}$ 
595 it becomes close to one or less than one when viscous forces condition flow in the fractured 596 block.

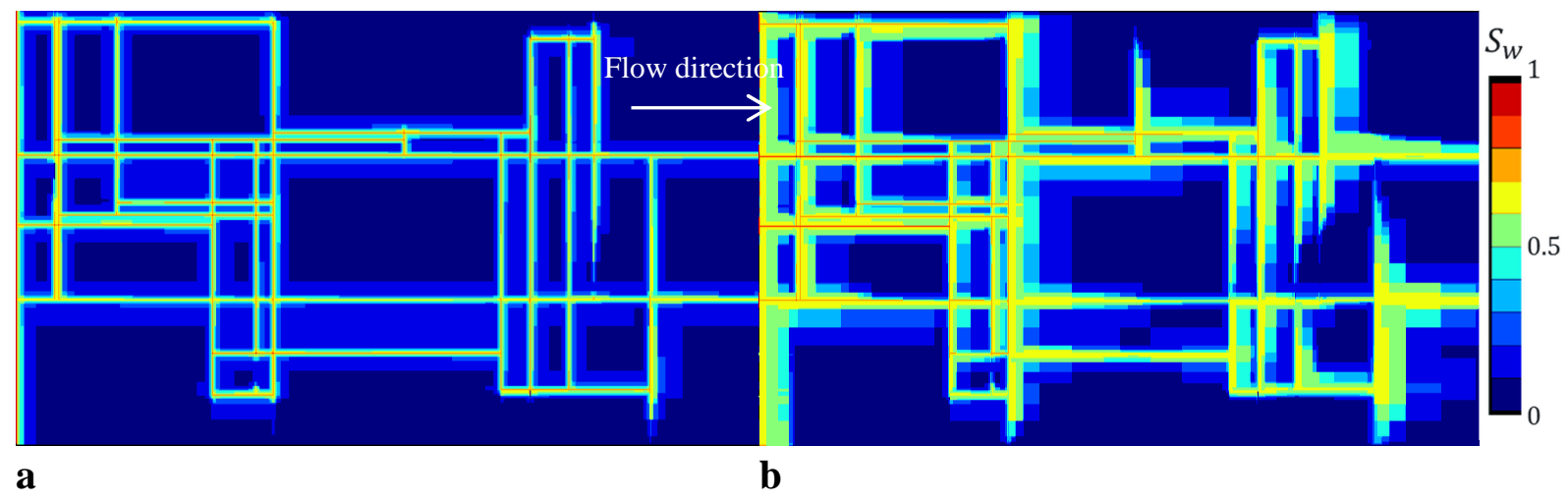

597 Fig. 8. Maps of water saturation in a water-flooding two-phase flow scenario. Calculations are performed over a

598 fine grid discretizing both the fracture network and matrix (system in Fig. 7). The system is initially saturated in 599 oil and water is injected in the fractures at a constant flow rate at the western boundary of the system. Oil 600 recovery is monitored at the eastern boundary (see Fig. 9). The fluid exchange between fractures and matrix is 601 dominated by capillary forces in map $a$ as both capillary and viscous forces are active in map $b$.

602

603

604

605

606

607

608

609

610

In the DFN approach where matrix-fracture exchanges are dominated by capillary effects $\left(n_{c}=4.1\right.$ with the settings of the simulations), water does not deeply invade the matrix (Fig 8.a,) while for the same injected water pore volume, high injection velocity and piston flow $\left(n_{c}=0.41\right)$ maintains higher water pressure gradients that help to a deeper water invasion of the matrix (Fig. 8.b). Calculations in the DFN serve as reference to the comparison of flow scenarios between dual porosity models assigned with matrix block size from the GI, EGI, MS and OBS methods (sizes of blocks are pictured in Fig. 7). The comparison is here performed by way of a single indicator defined as the evolution in time (precisely, the evolution with the water pore volume injected in the system) of the oil recovery ratio at the outlet of the fractured system. This oil recovery corresponds to the ratio of the cumulative volume of oil exiting the system to the total initial volume of oil in the system. This indicator is obviously macroscopic, with the meaning that it monitors the 
615 behavior of the system at the large scale (at least the homogenization scale of the fracture

616 network). It would not make sense to compare a local feature of the fracture network (e.g., the

617 pressure transients in a single fracture) with averaged behaviors obtained for the large blocks

618 (cells) of a dual porosity approach.

619
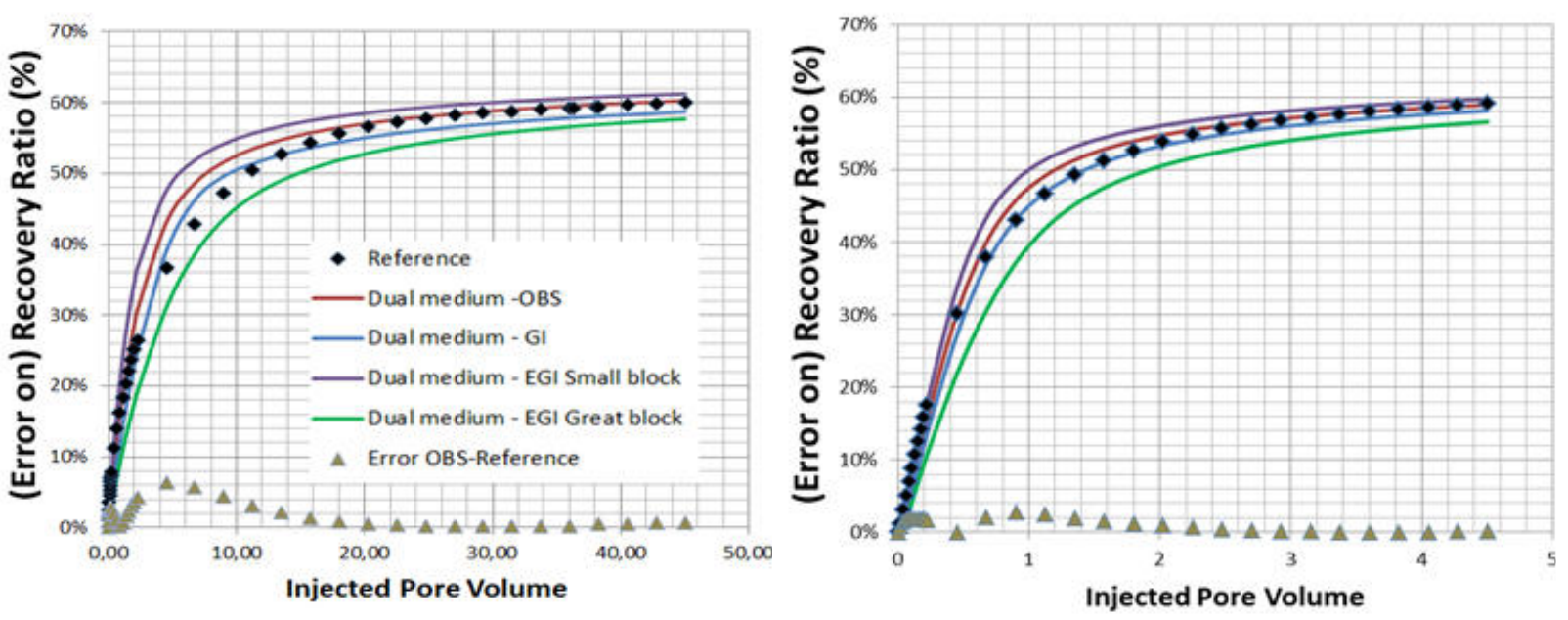

\section{$\mathbf{a}$}

b

620 Fig. 9. Oil recovery ratio versus water injected pore volumes at the eastern boundary of a fractured network (in

621 Fig. 7). The so-called reference is calculated by means of a finely discretized network as the other curves are drawn from a dual porosity model with various mean matrix block sizes. Results from the mean spacing technique for matrix block size evaluation are not reported because they are merged with those from the oriented block size technique. Capillary forces dominate the exchange rate between fractures and matrix in plot $a$, as both capillary and viscous forces are active in plot $b$.

Fig. 9 presents two plots of the oil recovery ratio as a function of the injected pore volume and stemming from flow scenarios with low and high injection velocities. The same oil recovery ratio of approximately $60 \%$ is reached for both flow scenarios, but with only 5 pore volumes in the case of high injection velocity compared with the 50 pore volumes 631 required by the case of low injection velocity. No dual porosity model with their different 632 matrix block size renders results that completely depart from the reference calculations in the 633 DFN. Since matrix block sizes calculated with OBS and MS techniques are quite similar (see 
634 Fig. 7 and Table 3), the results form dual porosity model simulations do not differ

635 significantly and only outputs from the OBS technique are reported in Fig. 9.

636 The OBS technique tends to slightly underestimate the matrix block size which

637 triggers a quicker oil extraction from the matrix and produces recovery curves slightly shifted

638 toward short injection times. The simulations handling the GI matrix blocks are also in very

639 good agreement with references, especially in the case of fracture-matrix exchanges enhanced

640 by high water injection rate. The matrix block sizes of EGI are still underestimated by the

641 "small block" measure and overestimated by the "large block" measure giving rise to

642 respectively faster and slower evolutions of the oil recovery ratio with respect to time. As

643 such, the EGI technique is not the most accurate to calculate matrix block sizes and should be

644 employed as a convenient way to provide minimal and maximal bounds to these sizes.

645 Notably, the fractured system discussed above does not significantly distinguish between GI

646 and OBS in terms of accuracy whichever the mechanism prevailing in fluid flux exchanges

647 between fractures and matrix. Nevertheless, we are reminded that the reference fracture

648 network was built to mitigate GI downsides. Fracture dead-ends were removed from the

649 network and the two fractures families were set parallel to the $x$ and $y$ directions of the

650 fractured block, thus allowing the GI method to infer a precise "invasion curve" $(A(X)$ in

651 Appendix B). This is why GI shows good performances in the present test cases as it

652 exhibited more discrepancies in the geometrical test cases discussed in Section 3.

653 At this stage, it must be raised that the OBS technique partly relies upon evaluations of

654 block-side properties such as fracture porosity and permeability, the latter being eventually

655 not representative of inner-block quantities when the portion of fractures intercepting the

656 block sides are not representative of the network geometry inside the block. To address the

657 eventual influence of this downside, we recalculated the two flow scenarios discussed above

658 for another fractured system (Fig. 10) which comprises a few long fractures located very close 
660 the boundaries of the system (those encircled in Fig 10) as the majority of matrix blocks 661 inside the system are rectangular with a ratio length to width barely exceeding a factor 3 . As

663 from that of the fractured "regular" system previously discussed.

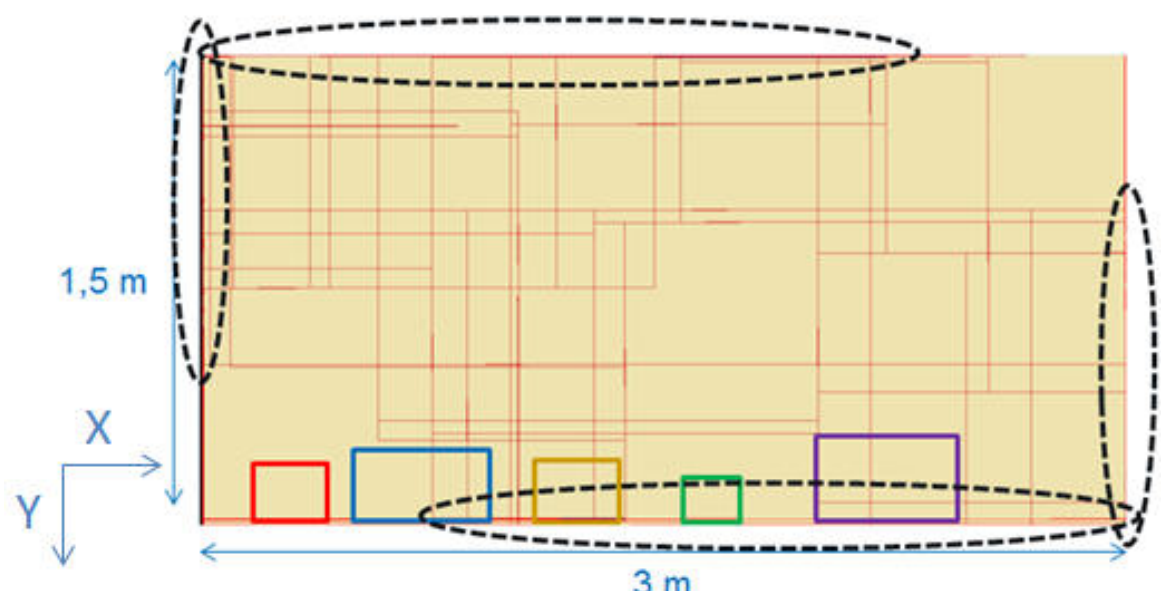

664

665

666

667

668

669

670

671
672

\begin{tabular}{|c|c|c|}
\hline & $x$ direction & $y$ direction \\
\hline$k_{f}\left[10^{-15} \mathrm{~m}^{2}\right]$ & 107.72 & 240 \\
\hline$k^{F N-S}\left[10^{-15} \mathrm{~m}^{2}\right]$ & 106.66 & 240 \\
\hline$\phi^{F N-S}[-]$ & 0.0106 & 0.024 \\
\hline$\phi_{f}[-]$ & 0.024 & \multicolumn{1}{|}{} \\
\hline
\end{tabular}

Fig. 10. Two-dimensional fracture network serving as a system finely discretized or handled as a dual-porosity model. Fractures close to the boundaries delimit very narrow matrix blocks (encircled) that depart from the shape of blocks within the fracture network. The identified sizes of matrix blocks in a dual porosity approach are reported as colored frames, from left to right: Red $=$ oriented block size method, Blue $=$ general imbibition method, Orange $=$ mean spacing imbibition method, Green and Purple $=$ small and large sizes from enhanced general imbibition method.

Table 4. Main macroscopic parameters of the fractured block in Fig. 10 to infer via the oriented block size technique the mean matrix block size of a dual porosity model. $k_{f}, \phi_{f}$ respectively are the permeability and porosity of the whole block, $k^{F N-S}, \phi^{F N-S}$ respectively are the permeability and porosity of the fracture network at the sides (normal to $\mathrm{x}$ and $\mathrm{y}$ directions) of the block. 


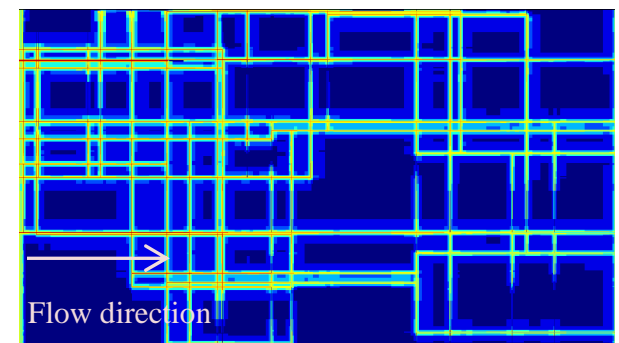

a

681

682

683

684

685

686

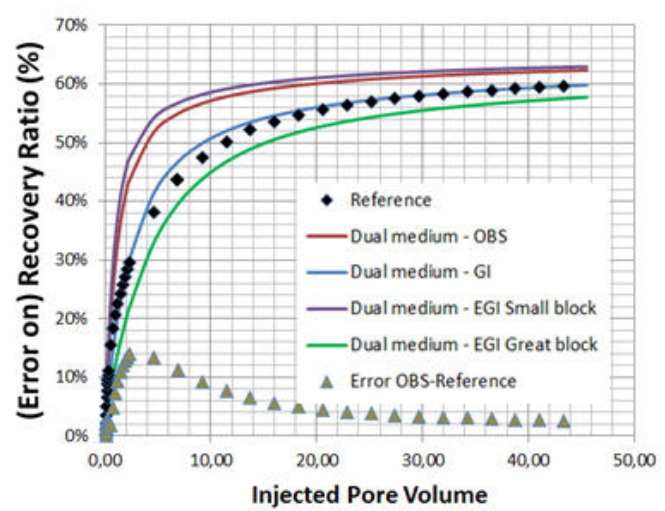

a

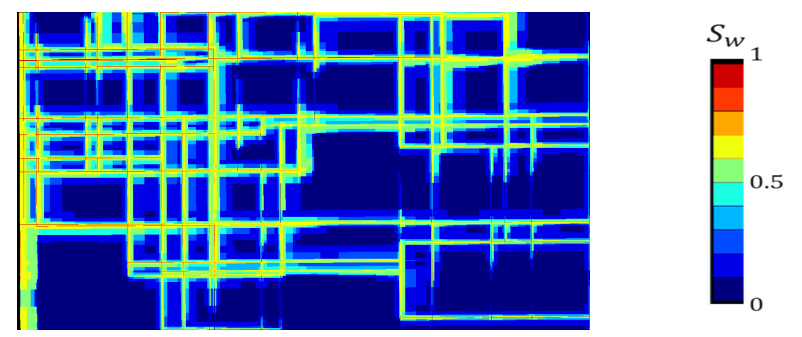

b

Fig. 11. Maps of water saturation in a water-flooding two-phase flow scenario. Calculations are performed over a fine grid discretizing both the fracture network and matrix (system in Fig. 10). The system is initially saturated in oil and water is injected in the fractures at a constant flow rate at the western boundary of the system. Oil recovery is monitored at the eastern boundary (see Fig. 12). The fluid exchange between fractures and matrix is dominated by capillary forces in map a as both capillary and viscous forces are active in map b.

687 Fig. 12. Oil recovery ratio versus water injected pore volumes at the eastern boundary of a fractured network (in

688 Fig. 10). The reference curve is calculated by means of a finely discretized network as the other curves are drawn 689 from a dual porosity model with various mean matrix block sizes. Results from the mean spacing technique are 690 not reported because they are merged with those from the oriented block size technique. Capillary forces 691 dominate the exchange rate between fractures and matrix in plot $a$, as both capillary and viscous forces are active in plot $b$. 
694 representation as a dual porosity system are changed much by the few fractures that do not 695 obey the general geometric and structural settings of the whole fractured block. This feature is 696 also evidenced by the comparison between the maps of water saturation in Fig. 8 and Fig. 11 697 that only differ by the locations of fractures underlined by high water saturations. However, 698 discrepancies between the reference (taken as the DFN) and the dual porosity approximations 699 increase. As for the preceding example, OBS and MS techniques provide very similar matrix 700 block sizes (these sizes are pictured in Fig. 10 and reported in Table 5) and similar dual701 porosity behaviors making that MS results are not discussed in the following.

\begin{tabular}{|c|c|c|c|c|c|}
\hline Block sizes & OBS & GI & MS & $\begin{array}{c}\text { EGI- } \\
\text { large }\end{array}$ & $\begin{array}{c}\text { EGI- } \\
\text { small }\end{array}$ \\
\hline$s_{x}[\mathrm{~m}]$ & 0.185 & 0.44 & 0.24 & 0.47 & 0.147 \\
\hline$s_{y}[\mathrm{~m}]$ & 0.149 & 0.21 & 0.19 & 0.325 & 0.138 \\
\hline
\end{tabular}

Table 5. Mean matrix block sizes of a dual porosity model as a surrogate to the discrete 705 fracture network in Fig. 7. the OBS technique overestimates the leaching of matrix block ( and oil production at the outlet of the system) because the smallest matrix block size (here along the $y$ direction) is underestimated. Whichever the algebraic form chosen in OBS to infer the matrix block size

711 (See Section 2), the method is in essence sensitive to fracture densities close to the boundaries 712 of the whole fractured block, either in regard of porosities at the sides of the block or of 713 permeability values in a "permeameter" type system. If the actual matrix block sizes close to 714 the boundaries of the block are smaller than inside the block, as is the case with the present example, the smallest matrix block size (here along $y$, see Table 5) is underestimated which 
favors rapid imbibition under capillary forces (see above the capillary number $n_{c}$ ) . Notably,

717 the GI technique is not sensitive to the few small matrix blocks of the DFN because it treats

718 the shell and inner parts of the block exactly the same way. For its part, the "small" EGI 719 technique underestimates the mean matrix block size as the "Large" EGI overestimates it 720 ("small" EGI overestimates matrix imbibition and "Large" EGI underestimates imbibition, see 721 oil recovery in Fig. 12a compared with reference).

When matrix-fracture exchanges occur as a conjunction of viscous and capillary forces 723 (see the capillary number in (17) and subsequently evaluated for DFN simulations), the OBS technique renders results the closest to reference. The key is that rapid water invasion of the fractured block through permeable fractures (see Fig. 11b) and subsequent viscous effects between matrix and fractures are dominated by percolation through the large fractures and their (large) neighbor matrix blocks. As the OBS technique identifies the correct largest matrix block size (here along the $x$ direction, see Table 5), flow simulations with a dual porosity model are convincing. This time, the GI technique underestimates oil recovery, as "Large" EGI does too, because the overestimated matrix block size (especially along the $x$ direction, see Table 5) is favorable to capillary imbibition but hampers water invasion along fractures and matrix block leaching at early injection times.

Finally, regarding performances in terms of computation costs, the different geometric methods were applied to a large DFN represented as a synthetic dual porosity reservoir of 1.05 million grid cells. For OBS and MS methods, matrix block size calculations were performed for each elementary cell and duplicated over all cells of the reservoir with total CPU times coming up as: $230 \mathrm{~s}$ for OBS and $1120 \mathrm{~s}$ for MS. Notably, the time counted for GI and EGI is that of calculations over a limited number of cells "strategically" sampled in the

739 whole grid of the dual porosity reservoir, yielding a fair representation of the system after $7401800 \mathrm{~s}$ of calculation. With approximately $4 \mathrm{~s}$ of calculation per cell and $10^{6}$ cells, identifying 
741 a matrix block size for each cell with GI and EGI methods would render impracticable

742 evaluations exceeding 45 days. When applied to known DFNs, both OBS and MS require a

743 pre-evaluation of the diagonal permeability tensor of the fractured block; by construction for

744 OBS (see Section 2) and to identify main flow directions in MS for which random lines

745 counting the spacing of fractures (see Appendix B) are parallel to these directions.

746 Differences of computations times between methods are in the straightforward (and fast)

747 application of an analytical solution for OBS opposed to the need for many random draws in 748 MS.

\section{Conclusions}

The Oriented Block Size (OBS) technique has been developed as a new way to infer

752 the mean matrix block sizes in porous fractured media with application to dual porosity 753 models of flow at the large scale. Matrix block sizes are calculated by seeking the equivalence 754 in terms of fracture permeability and fracture porosity between a fractured block and a 755 Warren and Root discrete fracture network made of three fracture families with regular 756 spacing and fracture planes normal to the main flow directions.

757 Two expressions of the OBS are available according to which type of fractured block 758 the method is applied. The first expression is well suited to infer matrix block sizes over 759 synthetic discrete fracture networks or well-known actual networks since it requires 760 identifying fracture porosity of the network, fracture porosity at the sides of the fractured 761 block, and the diagonal permeability tensor of the whole block (which can be calculated 762 analytically or numerically). This first expression is based on a rigorous algebraic 763 development which reveals precise and renders matrix block sizes close to expectations drawn 764 from various synthetic discrete fracture networks. The second expression is derived from the 765 first one via assumptions on the fracture porosities of the block. It has the advantage of being 
applicable to hardly accessible fracture network as encountered in the field. This second expression is compatible with an inference from field measurements such as hydraulic tests and observations in wells but should only render orders of magnitude instead of pinpoint values. Further works should address how matrix block sizes are influenced by uncertainty on available field data.

The OBS technique revealed much faster in terms of computation times compared with other available geometrical techniques developed to infer matrix block sizes. This feature is a promising avenue for tentative applications of the method in up-scaling the representation of huge fractured reservoirs as done for instance in the oil industry when optimization of oil recovery from various exploitation scenarios is planned. In this context, OBS and its precise evaluation of matrix block sizes is useful to the parameterization of dual porosity models for two phase flow either dominated by capillary forces or viscous forces. However, as the other methods, the OBS technique may fail in retrieving matrix block sizes within poorly connected 779 fracture networks. It is worth to note however that poorly connected networks are not valuable candidates to homogenization into a dual porosity model.

Finally, it must also be raised that OBS is associated with the identification of large 782 scale permeability tensors that are mostly sensitive to the backbone of a fractured network and 783 do not see fracture dead-ends. In the case of applications relying upon data from hydraulic 784 well tests, the type of occurring flow should be carefully considered. Two phase flow, mostly 785 witnessed by the propagation of an oil/water saturation front, will mainly record the effects of 786 the backbone, as single phase flow, mainly monitored by the transient evolution of water 787 pressure heads, would also be sensitive to dead-ends. It deserves some additional synthetic 788 test cases or confrontation to actual field data to see whether or not the OBS technique reveals 789 suited in these instances. 


\section{Acknowledgments}

792 The authors are grateful to IFPEN for funding the Ph-D fellowship of C. Jerbi. They are also

793 indebted to P. Delaplace at IFPEN for its fruitful advices provided all along this study.

794 


\section{fractured block and a Warren and Root (WR) block.}

We remind that an actual and well connected fractured block oriented with its main

798 directions along the main directions of flow indexed by $i=1,2,3$, can be characterized by mean

799 permeabilities $k_{i}^{F N-S}$ along the sides of the block as

800

$k_{i}^{F N-S}=\frac{1}{2\left(\Delta_{i+1} \Delta_{i+2}\right)}\left(\sum_{n=1}^{N f_{i-}} k_{n} l_{n} e_{n}+\sum_{n=1}^{N f_{i+}} k_{n} l_{n} e_{n}\right)$

801

The block size in direction $i$ is denoted $\Delta_{i}$ and the sides delimiting the block are also indexed

by $i$ but for limits normal to the main direction $i$. In addition, block sides are labelled $i-$ or

$803 i+$ according to their respective location upstream or downstream along direction $i$. In (A1),

$804 i$ is a cycling index such that, e.g., $i+1=3$ when $i=2$ and $i+1$ returns to 1 when $i=3$. The

805 sides $i-$ and $i+$ of the block are intercepted by a number of fractures $N f_{i-}$ and $N f_{i+}$, and $k$

806 is the local permeability of a fracture intercepting the side of the block over an apparent

807 length $l$ and with apparent fracture aperture $e$.

808 We also remind that a Warren and Root (WR) block concealing a regular fracture 809 network of three fracture families can be characterized by two expressions associating: 1- the 810 diagonal tensor of permeability of the whole block $k_{i}^{W R}(i=1,2,3), 2$ - the mean porosity of the

811 block $\phi^{W R}$, and 3 - the porosity of the block sides $\phi_{i}^{W R-S}(i=1,2,3)$, with the spacing $812 s_{i}(i=1,2,3)$, the aperture $e_{i}(i=1,2,3)$, and the local permeability $k_{i}(i=1,2,3)$ of the three 813 fracture families composing the WR block (for details, see Section 2). These expressions are

$814 \quad k_{i}=\frac{1}{2}\left(1+\frac{s_{i}}{e_{i}}\right) \sum_{j=1}^{3}(-1)^{\delta_{i, j}} k_{j}^{W R}$

$815 \quad k_{i}=\frac{1}{2\left(\phi^{W R}-\phi_{i}^{W R-S}\right)} \sum_{j=1}^{3}(-1)^{\delta_{i, j}} k_{j}^{W R}$ 
where $\delta_{i, j}$ is the Kronecker symbol, $\delta_{i, j}=1, i=j ; \delta_{i, j}=0, i \neq j$.

Following the idea that one can establish the equivalence between a WR network and

818 an actual fractured block regarding their hydraulic properties, it is assumed that $\phi^{W R}, \phi_{i}^{W R-S}$,

819 and $k_{i}^{W R}$ are similar to the equivalent properties in the actual fractured block, respectively

820 denoted as $\phi^{F N}, \phi_{i}^{F N-S}$, and $k_{i}^{F N-S}$ (see A1, for the latter). In the same vein, if a WR network

821 serves as reference for fixing model parameters of homogenized approaches to fractured

822 media, the characteristics of a WR network can be substituted by parameters of the

823 homogenized model. For example, the characteristics $s_{i}, e_{i}$, and $k_{j}^{W R}$ in (A2) are respectively

824 substituted by a mean matrix block size (also denoted $s_{i}$ as defined in (3)), a mean fracture

825 aperture $e_{f}$, and the entries of a diagonal tensor $\mathbf{k}_{j}^{f}$ of the homogenized model. With these

826 transformations, equating (A2) and (A3) results in

$$
\begin{aligned}
\left(1+\frac{s_{i}}{e_{f}}\right) \sum_{j=1}^{3}(-1)^{\delta_{i, j}} k_{j}^{f}=\frac{1}{\left(\phi^{F N}-\phi_{i}^{F N-S}\right)} \sum_{j=1}^{3}(-1)^{\delta_{i, j}} k_{j}^{F N-S} ; \text { i.e. }, \\
s_{i}=e_{f}\left(\frac{\sum_{j=1}^{3}(-1)^{\delta_{i, j}} k_{j}^{F N-S}}{\left(\phi^{F N}-\phi_{i}^{F N-S}\right) \sum_{j=1}^{3}(-1)^{\delta_{i, j}} k_{j}^{f}}-1\right)
\end{aligned}
$$

828 For the sake of simplification (see hereafter), the term -1 in the expression of $s_{i}$ can be

829 dropped by considering that the term in $\left(\phi^{F N}\right)^{-1}$ is much larger than one for usual fracture 830 porosity of a rock block barely exceeding a few percent. Stated differently, one might also 831 consider in (A4) that the matrix block size $s_{i}$ is much larger than the fracture aperture $e_{f}$ and 832 results in

$$
s_{i} \approx e_{f} \frac{\sum_{j=1}^{3}(-1)^{\delta_{i, j}} k_{j}^{F N-S}}{\left(\phi^{F N}-\phi_{i}^{F N-S}\right) \sum_{j=1}^{3}(-1)^{\delta_{i, j}} k_{j}^{f}}
$$


835 and the fracture permeability of a homogenized model $k_{j}^{f}$. It is noteworthy that $e_{f}$ is usually

836 not a parameter of a homogenized approach, and it makes sense to render (A5) (partly)

837 independent of any conjecture on the value of $e_{f}$. To this end, it is reasonably assumed that a

838 WR network has its matrix block sizes separating neighbor fractures independent of the 839 apertures $e_{i}$ of the fractures. Stated differently, it is assumed that a WR network with a 840 uniform aperture $e_{f}$ for its three fracture families can be found as equivalent to a WR with its 841 three fracture families with apertures $e_{i}$. With a uniform aperture $e_{f}$, a WR network would 842 render a value $\phi^{W R}-\phi_{i}^{W R-S}=e_{f} N f_{i} / \Delta_{i}$ with $N f_{i}$ the number of fractures in the family $i$, and

$843 \Delta_{i}$ the size of the whole fractured block along direction $i$. If the values $\phi^{F N}-\phi_{i}^{F N-S}$ were not 844 replacing their equivalent $\phi^{W R}-\phi_{i}^{W R-S}$ in Eq (A5), the latter would no longer depend on $e_{f}$.

845 Hence, our proposal is to calculate porosities of the actual fracture network by assigning the 846 whole skeleton of the network with a constant single-fracture aperture $e_{f}$. The fracture 847 network porosities for a constant aperture $e_{f}$ would write as

$\left.848 \quad\left(\phi^{F N}-\phi_{i}^{F N-S}\right)\right|_{e=e_{f}}=e_{f}\left(\phi^{F N^{*}}-\phi_{i}^{F N-S^{*}}\right)$

849 The terms $\phi^{F N^{*}}, \phi_{i}^{F N-S^{*}}\left[\mathrm{~L}^{-1}\right]$ denote porosities of the actual fracture skeleton per unit fracture 850 aperture (that can be calculated by assigning a uniform fracture aperture of 1 to the whole 851 fracture network). Substituting (A6) in (A5) simplifies the formulation of the matrix block 852 size into

$$
s_{i} \approx \frac{\sum_{j=1}^{3}(-1)^{\delta_{i, j}} k_{j}^{F N-S}}{\left(\phi^{F N^{*}}-\phi_{i}^{F N-S^{*}}\right) \sum_{j=1}^{3}(-1)^{\delta_{i, j}} k_{j}^{f}}
$$


857 facet permeability values of the actual fracture network $k_{j}^{F N-S}$, and 3- on structural properties

858 of the actual network resulting in fracture porosity values of the whole fractured block and its

859 sides $\phi^{F N^{*}}$ and $\phi_{i}^{F N-S^{*}}$, respectively. These features make that the form in (A7) is hardly

860 applicable to poorly-known natural systems and should be mainly used in problems dealing

861 with homogenization of systems with well-known geometry and discretization of synthetic

862 fracture networks and matrix blocks.

863 Nevertheless, another form of the mean matrix block size can be proposed. By

864 manipulating (A1), the permeability of the actual fracture network at the facets of the whole

865 fractured block can be rewritten as

866

$k_{i}^{F N-S}=\frac{\left(\sum_{n=1}^{N f_{i-}} l_{n} e_{n}+\sum_{n=1}^{N f_{i+}} l_{n} e_{n}\right)}{2\left(\Delta_{i+1} \Delta_{i+2}\right)} \times \frac{\left(\sum_{n=1}^{N f_{i-}} k_{n} l_{n} e_{n}+\sum_{n=1}^{N f_{i+}} k_{n} l_{n} e_{n}\right)}{\left(\sum_{n=1}^{N f_{i-}} l_{n} e_{n}+\sum_{n=1}^{N f_{i+}} l_{n} e_{n}\right)} ;$ i.e.

$$
k_{i}^{F N-S}=\phi_{i}^{F N-S} \bar{k}_{i} \quad \text { with } \quad \bar{k}_{i}=\frac{\left(\sum_{n=1}^{N f_{i-1}} k_{n} l_{n} e_{n}+\sum_{n=1}^{N f_{i+}} k_{n} l_{n} e_{n}\right)}{\left(\sum_{n=1}^{N f_{i-1}} l_{n} e_{n}+\sum_{n=1}^{N f_{i+1}} l_{n} e_{n}\right)}
$$

867 The tensor components $\bar{k}_{i}(i=1,2,3)$ in (A8) are an arithmetic mean of single-fracture 868 permeability values weighted by open fracture surface areas at the sides of the whole 869 fractured block. If we assume that these mean values are equal, irrespective of the facet of the 870 fractured block (which also can go with fractured systems candidates to homogenization), it 871 also means that the eventual anisotropy of permeability in the fracture network is just the 872 consequence of fractures densities normal to the flow directions, i.e., $k_{i}^{F N-S}=\phi_{i}^{F N-S} \bar{k}$. 873 Notably, this strong assumption stating that one can define a constant single-fracture 
874 permeability value $\bar{k}$ also goes with the existence of an equivalent uniform single-fracture 875 aperture $e_{f}$ for the whole fracture network. Reintroducing in (A5) the expression (A8) with a 876 constant value $\bar{k}$ and making use of rescaled porosities defined in (A6) as $\phi_{i}^{F N-S}=e_{f} \phi_{i}^{F N-S^{*}}$ 877 comes down to

$878 s_{i} \approx \frac{e_{f} \bar{k} \sum_{j=1}^{3}(-1)^{\delta_{i, j}} \phi_{j}^{F N-S^{*}}}{\left(\phi^{F N^{*}}-\phi_{i}^{F N-S^{*}}\right) \sum_{j=1}^{3}(-1)^{\delta_{i, j}} k_{j}^{f}}$

879 It can also be shown that a WR network with constant aperture $e_{f}$ for its three fracture 880 families has block and side porosities following the relation $\sum_{i} \phi_{i}^{W R-S^{*}}=2 \phi^{W R^{*}}$. If the rescaled 881 DFN is equivalent to the WR network, then one can state that $\sum_{i} \phi_{i}^{F N-S^{*}}=2 \phi^{F N^{*}}$. Noting that $882 \sum_{j=1}^{3}(-1)^{\delta_{i, j}} \phi_{j}^{F N-S^{*}}$ can also be rewritten as $\sum_{j=1}^{3} \phi_{j}^{F N-S^{*}}-2 \phi_{i}^{F N-S^{*}}$ and reintroducing the preceding 883 relationship between block and side porosities in (A9) results in

$884 s_{i} \approx \frac{2 e_{f} \bar{k}}{\sum_{j=1}^{3}(-1)^{\delta_{i, j}} k_{j}^{f}}$

885 In the case of field applications with poorly known and hardly accessible fracture 886 networks, (A10) returns the mean matrix block sizes in a fracture network based on the field 887 evaluations of the permeability tensor $\mathbf{k}^{f}$ of a whole fractured block, the average uniform 888 aperture $e_{f}$ and permeability $\bar{k}$ of a single fracture. Because the entries of (A10) are not 889 straightforward to obtain and may also be associated with important measurement errors, it is 890 expected that (A10) will only render orders of magnitude of mean matrix block sizes. 


\section{The geometrical imbibition (GI) method}

895

The method has been developed for two-dimensional flow models only. Three896 dimensional approaches are therefore handled as multilayer systems. For a two-dimensional 897 image of an actual or synthetic fracture network, the first task to handle consists in mapping 898 the image on a regular grid of square pixels. Each pixel is then assigned a value $d_{f}$ that 899 represents the distance between the center of the pixel and the closest fracture of the network. 900 One sums up the area of pixels whose distance $d_{f}$ is less than a prescribed value $X$, and the 901 area is then normalized by the total surface area of the image to form the quantity $A(X)$. The resulting measure $A(X)($ Fig. B1) is modeled as

$903 \quad A(X)=\frac{2 X}{a}+\frac{2 X}{b}-\frac{4 X^{2}}{a b}$

904 with $a$ and $b$ the resulting mean size of the matrix block of a two-dimensional dual porosity 905 model. $a$ and $b$ are obtained by minimizing the sum of squared errors between the model in 906 (B1) and the actual measures of $A(X)$.

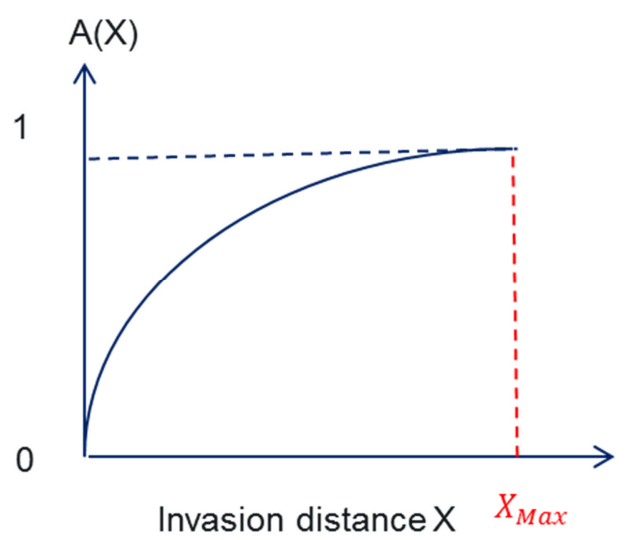

Fig. B1. General imbibition technique to mean matrix block size identification. Normalized invaded matrix area $A(X)$ as a function of the distance $X$ between a location in the matrix and the closest fracture. 
This method improves the two-dimensional GI technique by assuming that two mean matrix block sizes characterize the relationships between fractures and matrix. For locations in the matrix close to fractures, two types of matrix block interact with fractures, whereas locations far from fractures are influenced by a single size of matrix block. This feature makes that the quantity $A(X)$ drawn from the mapping of the actual fracture network (see above the

GI technique) is modeled by a discontinuous curve in the form

$$
\begin{aligned}
& A(X)=\alpha_{1}\left(\frac{2 X}{a_{1}}+\frac{2 X}{b_{1}}-\frac{4 X^{2}}{a_{1} b_{1}}\right)+\alpha_{2}\left(\frac{2 X}{a_{2}}+\frac{2 X}{b_{2}}-\frac{4 X^{2}}{a_{2} b_{2}}\right) ; X \leq \frac{a_{1}}{2} \\
& A(X)=\alpha_{2}\left(\frac{2 X}{a_{2}}+\frac{2 X}{b_{2}}-\frac{4 X^{2}}{a_{2} b_{2}}\right) ; X>\frac{a_{1}}{2}
\end{aligned}
$$

with $\left(a_{1}, b_{1}\right),\left(a_{2}, b_{2}\right)$ the size of the small and large matrix blocks respectively. $\alpha_{1}, \alpha_{2}$ are the

921 proportions of small (type 1) blocks and large (type 2) blocks with $\alpha_{2}=1-\alpha_{1}$. The distance

$922 X=a_{1} / 2$ is the threshold beyond which a single type of large matrix block is sufficient to 923 model interactions between fractures and matrix.

$$
A^{\prime}(X)=\frac{d A(X)}{d X}=\alpha_{1}\left(\frac{2}{a_{1}}+\frac{2}{b_{1}}-\frac{8 X}{a_{1} b_{1}}\right)+\alpha_{2}\left(\frac{2}{a_{2}}+\frac{2}{b_{2}}-\frac{8 X}{a_{2} b_{2}}\right) ; X \leq \frac{a_{1}}{2}
$$

$$
A^{\prime}(X)=\frac{d A(X)}{d X}=\alpha_{2}\left(\frac{2}{a_{2}}+\frac{2}{b_{2}}-\frac{8 X}{a_{2} b_{2}}\right) ; X>\frac{a_{1}}{2}
$$

929 This derivative appears as a decreasing piece-wise linear function of $X$ which can be fitted by 930 hand or numerically on the plot of actual values $A^{\prime}(X)$ (see Fig B2). The parameter $a_{1}$ is set 
932 of actual data. The parameter $a_{2}$ is defined as the length (distance) for which $A^{\prime}\left(a_{2} / 2\right)=0$

933 (see Fig. B2).

934

935 Fig. B2. Enhanced general imbibition technique to mean matrix block size evaluation. First-order derivative of the normalized invaded matrix area $A(X)$ as a function of the distance $X$ between a location in the matrix and the closest fracture. The derivative with respect to $X$ is modelled as a piecewise linear function allowing to infer a small and a large matrix block size.

939

$$
A^{\prime \prime}{ }_{1}=\frac{d A^{\prime}(X)}{d X}=-\frac{8 \alpha_{1}}{a_{1} b_{1}}-\frac{8 \alpha_{2}}{a_{2} b_{2}} ; X \leq \frac{a_{1}}{2}
$$

$$
A^{\prime \prime}=\frac{d A^{\prime}(X)}{d X}=-\frac{8 \alpha_{2}}{a_{2} b_{2}} ; X>\frac{a_{1}}{2}
$$

942 The difference of slopes on a plot of $A^{\prime}(X)$ can be identified with the expression of

$943 A^{\prime \prime}{ }_{1}-A^{\prime \prime}{ }_{2}=-8 \alpha_{1} / a_{1} b_{1}$ which in turn fixes the ratio $\alpha_{1} / b_{1}$ since $a_{1}$ has been previously 944 prescribed.

$$
\delta A^{\prime}=A^{\prime}\left(\left(a_{1} / 2\right)^{-}\right)-A^{\prime}\left(\left(a_{1} / 2\right)^{+}\right)=\frac{2}{\alpha_{1}}\left(\frac{1}{a_{1}}-\frac{1}{b_{1}}\right)
$$


947 Identifying (B5) with the value of the plot and associating the result with the identified value

$948 A^{\prime \prime}{ }_{1}(X)-A^{\prime \prime}{ }_{2}(X)$ renders two equations allowing for the calculation of both $\alpha_{1}$ and $b_{1}$

949 values.

950 Finally, the expression of $A^{\prime}(X)$ in $X=0$ which writes as

951

$A^{\prime}(0)=\alpha_{1}\left(\frac{2}{a_{1}}+\frac{2}{b_{1}}\right)+\left(1-\alpha_{1}\right)\left(\frac{2}{a_{2}}+\frac{2}{b_{2}}\right)$

952 is identified via the equivalent value observed on the plot of actual data (Fig. B2) and returns

953 the value of $b_{2}$.

954

955

The mean spacing (MS) technique

956

The principle of MS is sketched in Fig. B3.

957

958

959

960

961

962

963

964

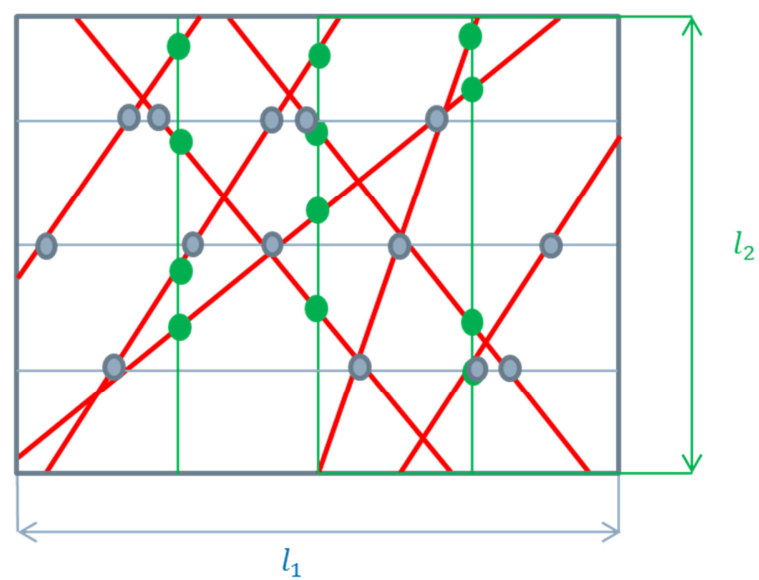

Fig. B3. Mean spacing technique to mean matrix block size evaluation. Distances between neighbor fractures are measured via the intersections between the fracture network and random lines parallel to the main directions of the fractured block.

For each main direction $i$ of a fractured block with length $l_{i}$, random lines parallel to direction $i$ and crossing the whole block are drawn. For each line, one counts as $n_{i}$ the number of intersections between the line and any fracture plane (or trace in a two-dimensional problem) of the fracture network. For each random line in the direction $i$, the mean distance 
965 between two successive intersections is $l_{i} /\left(n_{i}+1\right)$. The mean size of the matrix block in the 966 direction $i$ is defined as

$967 \quad s_{i}=l_{i}\left\langle\frac{1}{n_{i}+1}\right\rangle$

968 where averaging \langle\rangle is conducted over the whole set of random lines in the direction $i$. 
970

971 Adler, P.M., Mourzenko, V.V., Thovert, J-F., Bogdanov, I., 2005. Study of single and 972 multiphase flow in fractured porous media, using a percolation approach, Dynamics of Fluids 973 and Transport in Fractured Rocks, Geoph. Monog. Series, 162, 33-41.

974

975

976

977

978

979

980

981

982 homogeneous liquids in fissured rocks. J. Appl. Math. 24, 1286-1303.

983

984 985

986

987

988

989

990 Bourbiaux, B., 2010. Fractured reservoir simulation: a challenging and rewarding issue. Oil

991 Gas Sci. Technol. 65, 227-238.

992

993 Brooks, R.H., Corey, A.T., 1964. Hydraulic properties of porous media: Hydrology papers,

994 Colorado State University. 3. 
996 Coats, K.H., 1989. Implicit compositional simulation of single-porosity and dual porosity 997 reservoirs. SPE -18427-MS.

998

999 De Dreuzy, J. R., Rapaport, A., Babey, T., Harmand, J. , 2013. Influence of porosity 1000 structures on mixing-induced reactivity at chemical equilibrium in mobile/immobile Multi1001 Rate Mass Transfer (MRMT) and Multiple INteracting Continua (MINC) models. Water 1002 Resour. Res. 49(12), 8511-8530.

1003

1004 Folch, R., Casademunt, J., Hernandez-Machado, A., Ramirez-Piscina, L., 1999. Phase-Field 1005 model for Hele Shaw flows in arbitrary viscosity contrasts. I- Theoretical approach. Phys. 1006 Rev. E 60(2), 1724-1733.

1007

1008 Fourno, A, Grenier, C., Benabderrahmane, H., Delay, F., 2013. A continuum voxel approach 1009 to model flow in 3D fault networks: A new way to obtain up-scaled hydraulic conductivity 1010 tensors of grid cells. J. Hydrol. 493, 68-80.

1011

1012 Jourdain, X., Colliat, J.B., De Sa, C., Benboudjema, F., Gatuingt, F., 2014. Upscaling 1013 permeability for fractured concrete: meso-macro numerical approach coupled to strong 1014 discontinuities. Int. J. Numer. Anal. Met. 38(5), 536-550.

1015

1016 Karimi-Fard, M., Gong, B., Durlofsky, L.J., 2006. Generation of coarse-scale continuous flow 1017 models from detailed fracture characterization. Water Resour. Res. 42, W10423. 
1019 Kazemi, H., Merrill, L.S., Porterfield, K.L., Zeman, P.R., 1976. Numerical simulation of 1020 water-oil flow in naturally fractured reservoirs. SPE Reserv. Eval. Eng. 11(4), 750-758.

1021

1022 Landereau, P., Noetinger, B., Quintard, M., 2001. Quasi-steady two-equation models for 1023 diffusive transport in fractured porous media large-scale properties for densely fractured 1024 systems. Adv. Water Resour. 24(8), 863-876.

1025

Lemonnier, P., Bourbiaux, B., 2010a. Simulation of naturally fractured reservoirs. State of the art, part 1, Phyiscal mechanisms and simulator formulation. Oil Gas Sci.Technol. 65(2), 239262.

1029

1030 Lemonnier, P., Bourbiaux, B., 2010b. Simulation of naturally fractured reservoirs. State of the 1031 art, part 2, Matrix-fracture transfers and typical features of numerical studies. Oil Gas Sci. 1032 Technol. 65(2), 263-286.

1033

1034 Lim, K.T., Aziz, K., 1995. Matrix-fracture transfer shape factors for dual porosity simulators. 1035 J. Petrol. Sci. Eng. 13, 169-178.

1036

1037 Long, J.C.S., Remer, J.S., Wilson, C.R., Witherspoon, P.A., 1982. Porous medium equivalent 1038 for networks of discontinuous fractures. Water Resour. Res. 18(13), 645-658.

1039

1040 Matthai, S.K., Nick, H.M., 2009. Upscaling two-phase flow in naturally fractured reservoirs. 1041 The AAPG Bull. 93(11), 1621-1632. 
1043 Narr, W., 1996. Estimating average fracture spacing in subsurface rock. The AAPG Bull.

1044 80(10), 1565-1586.

1045

1046 Neuman, S.P., 1988. A proposed conceptual framework and methodology for investigating 1047 flow and transport in Swedish crystalline rock. SKB Swedish Nuclear Fuel and Waste 1048 Management Co., Stockholm, September, Arbetsrapport.

1049

1050 Noetinger, B., Estébenet, T., 2000. Up-scaling double porosity fractured media using 1051 contineous-time random walks method. Transport Porous Med. 39(13), 315-337.

1052

1053 Noetinger B., Estebenet, T., Landereau, P., 2001. A direct determination of the transient 1054 exchange term of fractured media using a continuous time random walk method. Transport 1055 Porous Med. 44, 539-557.

1056

1057 Noetinger, B., Jarrige, N., 2012. A quasi steady state method for solving transient Darcy flow 1058 in complex 3D fractured networks. J. Comput. Phys. 231, 23-38.

1059

1060 Oda, M., 1985. Geologic analysis of naturally fractured reservoirs. Geotechnique. 35, 4831061495.

1062

1063 Park, C.M., Homsy, G.M., 1984. Two-phase displacement in Hele Shaw cells. Theory. J. 1064 Fluid Mech. 139, 291-398.

1065

1066 Pruess, K., Narasimhan, T.N., 1985. A practical method for modeling fluid and heat flow in 1067 fractured porous media. SPE J. 25(1), 14-26. 
1069 Pruess, K. Wang, J.S.Y., Tsang, Y.W., 1990. On thermohydrologic conditions near high-level 1070 nuclear wastes emplaced in partially saturated fractured tuff. 2- Effective continuum 1071 approximation. Water Resour. Res. 26(6), 1249-1261.

1072

1073 Quintard, M., Whitaker, S., 1993. One and two-equation models for transient diffusion 1074 processes in two-phase systems. Adv. Heat Transf. 23, 369-465.

1075

1076 Quintard, M., Whitaker, S., 1996. Transport in chemically and mechanically heterogeneous 1077 porous media. Adv. Water Resour. 19, 29-60.

1078

1079 Tatomir, A.B., Szykiewicz, A., Class, H., Helming, R., 2011. Modeling two phase flow in 1080 large scale fractured porous media with an extended multiple interacting continua. CMES $108177(2), 81-112$.

1082

1083 Thomas, L.K., Dixon, T.N., Pierson, R.G., 1983. Fractured reservoir simulation. SPE J. 1084 23(11), 42-54.

1085

1086 Ueda, Y., Murata, S., Watanabe, Y., Funats, K., 1989. Investigation of the shape factor used 1087 in the dual-porosity reservoir simulator. SPE-19469-MS.

1088

1089 Unsal, E., Matthäi, S.K., Blunt, M.J., 2010. Simulation of multiphase flow in fractured 1090 reservoirs using a fracture-only model with transfer functions. Comput. Geosci. 14, 527-538. 1091 
1092 Warren, J.E., Root, P.J., 1963. The behavior of naturally fractured reservoirs. SPE J. 3, 245-

1093255. 